Contribution to the proceedings of conference "RG 2000" (Taxco, Mexico, Jan. 1999). To be published in Phys. Repts;

JINR preprint E2-2000-9

\title{
BOGOLIUBOV Renormalization Group and Symmetry of Solution in Mathematical Physics
}

\author{
Dmitrij V. Shirkov \\ Bogoliubov Laboratory, JINR, Dubna, 141980 Russia; e-mail: shirkovd@thsun1.jinr.ru \\ and \\ Vladimir F. Kovalev \\ Institute for Mathematical Modelling, Moscow,125047 Russia; e-mail: kovalev@imamod.ru
}

\begin{abstract}
Evolution of the concept known in the theoretical physics as the Renormalization Group (RG) is presented. The corresponding symmetry, that has been first introduced in QFT in mid-fifties, is a continuous symmetry of a solution with respect to transformation involving parameters (e.g., of boundary condition) specifying some particular solution.

After short detour into Wilson's discrete semi-group, we follow the expansion of QFT RG and argue that the underlying transformation, being considered as a reparameterisation one, is closely related to the self-similarity property. It can be treated as its generalization, the Functional Self-similarity (FS).

Then, we review the essential progress during the last decade of the FS concept in application to boundary value problem formulated in terms of differential equations. A summary of a regular approach recently devised for discovering the RG $=$ FS symmetries with the help of the modern Lie group analysis and some of its applications are given.

As a main physical illustration, we give application of new approach to solution for a problem of self-focusing laser beam in a non-linear medium.
\end{abstract}




\section{Contents}

$\begin{array}{lll}1 & \text { The Bogoliubov renormalization group } & 3\end{array}$

1.1 Historical introduction $\ldots \ldots \ldots \ldots$. . . . . . . . . . . . . . . . . . . . . . . .

1.1 .1 The discovery of the renormalization groun . . . . . . . . . . . . . . . . . . . . . . . . . . . . . . .

$1.1 .2 \quad$ Creation of the RG method . . . . . . . . . . . . . . . . . . . . . . . 4

1.2 The Bogoliubov RG: Symmetry of a solution . . . . . . . . . . . . . . 5

1.3 Renorm-group method . . . . . . . . . . . . . . . . . . . . 7

1.3 .1 The algorithm . . . . . . . . . . . . . . . . . . . . . 7

1.3 .2 Simple illustration . . . . . . . . . . . . . . . . . . . . . . . . 8

$1.3 .3 \quad$ RGM usage in QFT . . . . . . . . . . . . . . . . . . . . . . . . . . . . 9

2 Evolution of Renormalization Group $\quad 9$

2.1 Renormalization Group Evolving . . . . . . . . . . . . . . . . . . . . . . . 9

2.1.1 Kadanoff-Wilson RG in critical phenomena . . . . . . . . . . . . . . . 10

2.1.2 Bogoliubov symmetry outside QFT . . . . . . . . . . . . . . . . . . . . 12

2.2 Difference between Bogoliubov RG and KW-RG . . . . . . . . . . . . . . 14

2.3 Functional self-similarity . . . . . . . . . . . . . . . . . . . . . . . . . . . . 14

3 Symmetry of solution in Mathematical Physics 16

3.1 Constructing RG-symmetries and their use . . . . . . . . . . . . . . . . . . 16

3.2 Examples of solution improving . . . . . . . . . . . . . . . . . . . . . . 21

3.2 .1 Modified Burgers equation . . . . . . . . . . . . . . . . . . . . . 21

3.2.2 BVP for ODE: simple example . . . . . . . . . . . . . . . . . . . . . 23

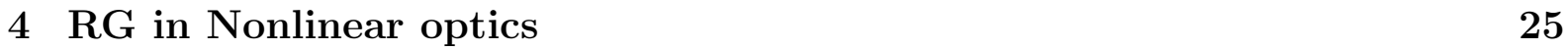

4.1 Formulation of a problem . . . . . . . . . . . . . . . . . . . . 25

4.2 Plane geometry . . . . . . . . . . . . . . . . . . . . . . . 26

4.3 Cylindrical geometry . . . . . . . . . . . . . . . . . . . . . . . 28

$\begin{array}{lll}5 & \text { Overview } & 30\end{array}$

$\begin{array}{ll}\text { References } & 33\end{array}$ 


\section{The Bogoliubov renormalization group}

\subsection{Historical introduction}

\subsubsection{The discovery of the renormalization group}

In 1952-1953 Stückelberg and Petermann [1] discovered formations related to finite arbitrariness arising in $S$-matrix elements upon elimination of ultraviolet (UV) divergences. These authors used the notion of normalization group as a Lie transformation group generated by differential operators connected with renormalization of the coupling constant $e$.

In the following year, on the basis of (infinite) Dyson's renormalization transformations formulated in the regularized form, Gell-Mann and Low [3] derived functional equations (FEs) for the QED propagators in the UV limit. The appendix to this article contained the general solution (obtained by T.D. Lee) of FE for the renormalized transverse photon propagator amplitude $d\left(Q^{2} / \lambda^{2}, e^{2}\right)$ ( $\lambda$ - cutoff defined as a normalization momentum). This solution was used for a qualitative analysis of the quantum electromagnetic interaction behaviour at small distances. Two possibilities, namely, infinite and finite charge renormalizations were pointed out. However, paper [3] paid no attention to the group character of the analysis and of the qualitative results obtained there. The authors missed a chance to establish a connection between their results and the QED perturbation theory and did not discuss the possibility that a ghost pole solution might exist.

The decisive step was made by Bogoliubov and the present author [4, 6, 6] in 1955 [ . Using the group properties of finite Dyson transformations for the coupling constant, fields and Green functions, they derived functional group equations for the renormalized propagators and vertices in QED in the general (i.e., with the electron mass taken into account) case.

In the modern notation, the first equation

$$
\bar{\alpha}(x, y ; \alpha)=\bar{\alpha}\left(\frac{x}{t}, \frac{y}{t} ; \bar{\alpha}(t, y ; \alpha)\right) ; x=\frac{Q^{2}}{\mu^{2}}, \quad y=\frac{m^{2}}{\mu^{2}}
$$

is that for the invariant charge (now widely known also as an effective or running coupling) $\bar{\alpha}=\alpha d\left(x, y ; \alpha=e^{2}\right)$ and the second -

$$
s(x, y ; \alpha)=s(t, y ; \alpha) s\left(\frac{x}{t}, \frac{y}{t} ; \bar{\alpha}(t, y ; \alpha)\right)
$$

- for the electron propagator amplitude.

These equations obey a remarkable property: the product $e^{2} d \equiv \bar{\alpha}$ of the electron charge squared and the photon transverse propagator amplitude enters into both FEs.

\footnotetext{
${ }^{1}$ For a more detailed exposition of the RG early history, see our recent reviews [2].

${ }^{2}$ See also two survey papers [7] published in English in 1956.
} 
This product is invariant with respect to finite Dyson's transformation (as it is stated by Eq.(1)) which now can be written in the form

$$
R_{t}:\left\{\mu^{2} \rightarrow t \mu^{2}, \alpha \rightarrow \bar{\alpha}(t, y ; \alpha)\right\}
$$

We called this product invariant charge and introduced the term renormalization group.

Let us emphasize that, unlike in Refs. [1], 3], in the Bogoliubov formulation there is no reference to UV divergences and their subtraction or regularization. At the same time, technically, there is no simplification due to the massless nature of the UV asymptotics. Here, the homogeneity of the transfer momentum scale $Q$ is explicitly violated by the mass $m$. Nevertheless, the symmetry with respect to transformation $R_{t}$ (even though a bit more involved) underlying RG is formulated as an exact property of the solution. This is what we mean when using the term Bogoliubov renormalization group or renormgroup for short.

The differential Lie equations for $\bar{\alpha}$ and for the electron propagator

$$
\frac{\partial \bar{\alpha}(x, y ; \alpha)}{\partial \ln x}=\beta\left(\frac{y}{x}, \bar{\alpha}(x, y ; \alpha)\right) ; \quad \frac{\partial s(x, y ; \alpha)}{\partial \ln x}=\gamma\left(\frac{y}{x}, \bar{\alpha}(x, y ; \alpha)\right) s(x, y ; \alpha),
$$

with

$$
\beta(y, \alpha)=\frac{\partial \bar{\alpha}(\xi, y ; \alpha)}{\partial \xi}, \quad \gamma(y, \alpha)=\frac{\partial s(\xi, y ; \alpha)}{\partial \xi} \quad \text { at } \xi=1
$$

were first derived in [4] by differentiating FEs (11) and (2) over $x$ at the point $t=x$. On the other hand, by differentiating the same equations over $t$ one obtains [8]

$$
X \bar{\alpha}(x, y ; \alpha)=0 ; \quad X s(x, y ; \alpha)=\gamma(y, \alpha) s(x, y ; \alpha)
$$

with

$$
X=x \partial_{x}+y \partial_{y}-\beta(y, \alpha) \partial_{\alpha} \quad\left(\partial_{x} \equiv \partial / \partial x\right),
$$

the Lie infinitesimal operator.

\subsubsection{Creation of the $R G$ method}

Another important achievement of [4] consisted in formulating a simple algorithm for improving an approximate perturbative solution by combining it with Lie group equations - for detail, see below Section 1.3.

In our adjacent publication [5] this algorithm was effectively used to analyse the UV and infrared (IR) behaviour in QED. In particular, the one-loop UV asymptotics of the photon propagator as well as the IR behavior of the electron propagator in the transverse gauge

$$
\bar{\alpha}_{\mathrm{rg}}^{(1)}(x ; \alpha)=\frac{\alpha}{1-\frac{\alpha}{3 \pi} \ln x} \quad, \quad s(x, y ; \alpha) \approx\left(p^{2} / m^{2}-1\right)^{-3 \alpha / 2 \pi}
$$


were derived. At that time, these expressions, summing the leading log's terms were already known from papers by Landau with collaborators [9]. However, Landau's approach did not provide a means for constructing subsequent approximations.

The simple technique for calculating higher approximations was found only within the new renormgroup method. In the same paper, starting with the next order perturbation expression $\bar{\alpha}_{\mathrm{pt}}^{(2)}(x ; \alpha)$ containing the $\alpha^{3} \ln x$ term, we arrived at the second renormgroup approximation (see below Section 1.3.2)

$$
\bar{\alpha}_{\mathrm{rg}}^{(2)}(x ; \alpha)=\frac{\alpha}{1-\frac{\alpha}{3 \pi} \ln x+\frac{3 \alpha}{4 \pi} \ln \left(1-\frac{\alpha}{3 \pi} \ln x\right)}
$$

which performs infinite summation of the $\alpha^{2}(\alpha \ln )^{n}$ terms. This two-loop solution for invariant coupling first obtained in [5] contains the nontrivial log-of-log dependence which is now widely known of the "next-to-leading logs" approximation for the running coupling in quantum chromodynamics (QCD) - see, below, Eq.(21).

Comparing (9) with (8), one concludes that two-loop correction is essential in the vicinity of the ghost pole at $x_{1}=\exp (3 \pi / \alpha)$. This also shows that the RG method is a regular procedure, within which it is easy to estimate the range of applicability of its results.

Quite soon, this approach was formulated [6] for the case of QFT with two coupling constants. To the system of FEs for two invariant couplings there corresponds a coupled system of nonlinear differential equations (DEs). The last was used [10 to study the UV behavior of the $\pi-N$ interaction at the one-loop level.

Thus, in Refs. [4, 5, 6] and [10] RG was directly connected with practical computations of the UV and IR asymptotics. Since then, this technique, the renormalization group method (RGM) ? has become the sole means of asymptotic analysis in local QFT.

\subsection{The Bogoliubov RG: Symmetry of a solution}

The RG transformation. Generally, RG can be defined as a continuous one-parameter group of specific transformations of a partial solution (or the solution characteristic) of a problem, a solution that is fixed by a boundary condition. The RG transformation involves boundary condition parameters and corresponds to some change in the way of imposing this condition.

For illustration, imagine one-argument solution characteristic $f(x)$ that has to be specified by the boundary condition $f\left(x_{0}\right)=f_{0}$. Formally, represent a given characteristic of a partial solution as a function of boundary parameters as well: $f(x)=f\left(x, x_{0}, f_{0}\right)$. This step can be treated as an embedding operation. Without loss of generality $f$ can be written in a form of a two-argument function $F\left(x / x_{0}, f_{0}\right)$ with the property $F(1, \gamma)=\gamma$.

The RG transformation then corresponds to a changeover of the way of parameterization, say from $\left\{x_{0}, f_{0}\right\}$ to $\left\{x_{1}, f_{1}\right\}$ for the same solution. In other words, the $x$ argument

\footnotetext{
${ }^{3}$ Being summarized in the special chapter of the first edition of monograph [1].
} 
value, at which the boundary condition is given, can be changed for $x_{1}$ with $f\left(x_{1}\right)=f_{1}$. The equality $F\left(x / x_{0}, f_{0}\right)=F\left(x / x_{1}, f_{1}\right)$ now reflects the fact that under such a change the form of the function $F$ itself is not modified. Noting that $f_{1}=F\left(x_{1} / x_{0}, f_{0}\right)$, we get

$$
F\left(\xi, f_{0}\right)=F\left(\xi / t, F\left(t, f_{0}\right)\right) ; \xi=x / x_{0}, t=x_{1} / x_{0}
$$

The group transformation here is $\left\{\xi \rightarrow \xi / t, \quad f_{0} \rightarrow F\left(t, f_{0}\right)\right\}$.

The renormgroup transformation for a given solution of some physical problem in the simplest case can now be defined as

a simultaneous one-parameter transformation of two variables, say $x$ and $g$, by

$$
R_{t}:\left\{x \rightarrow x^{\prime}=x / t, \quad g \rightarrow g^{\prime}=\bar{g}(t, g)\right\},
$$

the first being a scaling of a coordinate $x$ (or reference point) and the second - a more complicated functional transformation of the solution characteristic. The equation

$$
\bar{g}(x, g)=\bar{g}(x / t, \bar{g}(t, g))
$$

for the transformation function $\bar{g}$ provides the group property $T_{\tau t}=T_{\tau} T_{t}$ of the transformation (10).

They are just the RG FEs and transformation for a massless QFT model with one coupling constant $g$. In that case $x=Q^{2} / \mu^{2}$ is the ratio of a 4-momentum $Q$ squared to a "normalization" momentum $\mu$ squared and $g$, the coupling constant.

The RG transformation (10) of a QFT amplitude $s$ is of the form (compare with Eq.(2))

$$
R_{t} \cdot s(x, g) \equiv e^{-\ln t X} s(x, g)=s(x / t, \bar{g}(t, g))=z_{s}^{-1} s(x, g) ; \quad z_{s}=s(t, g) .
$$

Several generalizations are in order.

a. "Massive" case. For example, in QFT, if we do not neglect mass $m$ of a particle, we have to insert an additional dimensionless argument into the invariant coupling $\bar{g}$ which now has to be considered as a function of three variables: $x=Q^{2} / \mu^{2}, y=m^{2} / \mu^{2}$, and $g$. The presence of a new "mass" argument $y$ modifies the group transformation (10) and the FE (11)

$$
R_{t}:\left\{x^{\prime}=\frac{x}{t}, y^{\prime}=\frac{y}{t}, g^{\prime}=\bar{g}(t, y ; g)\right\} ; \bar{g}(x, y ; g)=\bar{g}\left(\frac{x}{t}, \frac{y}{t} ; \bar{g}(t, y ; g)\right) \text {. }
$$

Here, it is important that the new parameter $y$ (which, physically, should be close to the $x$ variable, as it scales similarly) enters also into the transformation law of $g$.

If the considered QFT model, like QCD, contains several masses, there will be several mass arguments $y \rightarrow\{y\} \equiv y_{1}, y_{2}, \ldots, y_{n}$. 
b. Multi-coupling case. A more involved generalization corresponds to transition to the case with several coupling constants: $g \rightarrow\{g\}=g_{1}, \ldots, g_{k}$. Here, there arise a "family" of effective couplings

$$
\bar{g} \rightarrow\{\bar{g}\}, \quad \bar{g}_{i}=\bar{g}_{i}(x, y ;\{g\}) ; \quad i=1,2, \ldots, k,
$$

satisfying the system of coupled functional equations

$$
\bar{g}_{i}(x, y ;\{g\})=\bar{g}_{i}(x / t, y / t ;\{\bar{g}(t, y ;\{g\})\}) .
$$

The RG transformation now is

$$
R_{t}:\{x \rightarrow x / t, y \rightarrow y / t, \quad\{g\} \rightarrow\{g(t)\}\}, \quad g_{i}(t)=\bar{g}_{i}(t, y ;\{g\}) .
$$

\subsection{Renorm-group method}

\subsubsection{The algorithm}

The idea of the approximate solution marriage [由, 5] to group symmetry can be realized with the help of group DEs. If we define $\beta$ and $\gamma$ (the so-called "generators" on physical slang) from some approximate solutions and then solve evolutional DEs, we obtain the $R G$ improved solutions that obey the group symmetry and correspond to the approximate solutions used as an input.

Now we can formulate an algorithm of improving an approximate solution. The procedure is given by the following prescription which we illustrate by a massless one-coupling case (因) and (5):

Assume some approximate solution $\bar{g}_{\text {appr }}(x, g), s_{\text {appr }}(x, g)$ is known.

1. On the basis of Eq.(5) define the beta- and gamma-functions

$$
\left.\beta(g) \stackrel{\text { def }}{=} \frac{\partial}{\partial \xi} \bar{g}_{\text {appr }}(\xi, g)\right|_{\xi=1} ;\left.\quad \gamma(g) \stackrel{\text { def }}{=} \frac{\partial}{\partial \xi} s_{\text {appr }}(\xi, g)\right|_{\xi=1} .
$$

2. Integrate the first of Eqs.(四), i.e., construct the function

$$
f(g) \stackrel{\text { def }}{=} \int^{g} \frac{d \tau}{\beta(\tau)},
$$

3. Resolve the obtained equation, i.e.,

$$
\bar{g}_{\mathrm{rg}}(x, g)=f^{-1}\{f(g)+\ln x\} .
$$

4. Integrate the second of Eqs.(体) using this expression $\bar{g}_{\mathrm{rg}}$ in its r.h.s. to obtain $s_{\mathrm{rg}}(x, g)$ in the explicit form.

5. Then, the expressions $\bar{g}_{\mathrm{rg}}$ and $s_{\mathrm{rg}}$ precisely satisfy the RG symmetry, i.e., they are exact solutions of Eqs.(11) and (12) corresponding to $\bar{g}_{\text {appr }}$ and $s_{\text {appr }}$ used as an input. 


\subsubsection{Simple illustration}

For illustration, take the simplest perturbative expression $\bar{g}_{\mathrm{pt}}^{(1)}=g-g^{2} \beta_{1} \ln x$ for $\bar{g}_{\mathrm{appr}}$ and $s_{\mathrm{pt}}^{(1)}=1-g \gamma_{1} \ln x$. Here, $\beta(g)=-\beta_{1} g^{2}, \gamma(g)=-\gamma_{1} g$ and integration of (4) gives explicit expressions

$$
\bar{g}_{\mathrm{rg}}^{(1)}(x, g)=\frac{g}{1+g \beta_{1} \ln x}, \quad s_{\mathrm{rg}}^{(1)}(x, g)=(\bar{g}(x, g) / g)^{\nu_{1}} ; \quad \nu_{1}=\gamma_{1} / \beta_{1},
$$

which, on the one hand, exactly satisfy the RG symmetry and, on the other, being expanded in powers of $g$, correlate with $\bar{g}_{\mathrm{pt}}$ and $s_{\mathrm{pt}}$.

Now, on the basis of geometric progression (20), let us present the 2-loop perturbative approximation for $\bar{g}$ in the form $\bar{g}_{\mathrm{pt}}^{(2)}=g-g^{2} \beta_{1} \ln x+g^{3}\left(\beta_{1}^{2} \ln ^{2} x-\beta_{2} \ln x\right)$. By using this expression as an input in Eq.(17), we have $\beta^{(2)}(g)=-\beta_{1} g^{2}-\beta_{2} g^{3}$ and then (step 2)

$$
\beta_{1} f^{(2)}(z)=-\int^{z} \frac{d \tau}{\tau^{2}+b \tau^{3}}=\frac{1}{z}+b \ln \frac{z}{1+b z} ; \quad b=\frac{\beta_{2}}{\beta_{1}} .
$$

To make the last step, we have to start with the equation

$$
f^{(2)}\left[\bar{g}_{\mathrm{rg}}^{(2)}(x, g)\right]=f^{(2)}(g)+\beta_{1} \ln x
$$

which is a transcendental one and has no simple explicit solutionf. Due to this, one usually resolves this relation approximately. Take into account that the second, logarithmic, contribution to $f^{(2)}(z)$ is a small correction to the first one at $b z \ll 1$. Under this reservation, we can substitute the one-loop RG expression (20) instead of $\bar{g}_{\mathrm{rg}}^{(2)}$ into this correction and obtain the explicit "iterative" solution

$$
\bar{g}_{\mathrm{rg}}^{(2)}=\frac{g}{1+g \beta_{1} l+g\left(\beta_{2} / \beta_{1}\right) \ln \left[1+g \beta_{1} l\right]} ; \quad l=\ln x .
$$

An analogous procedure for $s_{\mathrm{pt}}^{(2)}=1-g \gamma_{1} \ln x+g^{2}\left(\gamma_{1}\left(\gamma_{1}+1\right) \beta_{1}^{2} \ln ^{2} x-\gamma_{2} \ln x\right)$ yields

$$
s_{\mathrm{rg}}^{(2)}=\frac{S\left(\bar{g}_{\mathrm{rg}}^{(2)}(x, g)\right)}{S(g)} \quad \text { with } \quad S(g)=g^{\nu_{1}} e^{\nu_{2} g} \quad \text { and } \quad \nu_{2}=\frac{\beta_{1} \gamma_{2}-\gamma_{1} \beta_{2}}{\beta_{1}^{2}}
$$

These results are interesting from several aspects.

- First, being expanded in $g$ and $g l$ powers, they produce an infinite series containing "leading" and "next-to-leading" UV logarithmic contributions.

- Second, they contain a nontrivial analytic dependence $\ln \left(1+g \beta_{1} l\right) \sim \ln \left(\ln Q^{2}\right)$ which is absent in the perturbation input.

- Third, being compared with the one-loop solution, Eq.(20), they demonstrate an algorithm of subsequent improving of accuracy, i.e., of RGM regularity.

\footnotetext{
${ }^{4}$ It can be expressed via special, Lambert, $W$-function: $W(z) \exp ^{W(z)}=z$; see, e.g., Ref.[12].
} 


\subsubsection{RGM usage in $Q F T$}

As we have seen, QFT perturbation expression of finite order does not obey the RG symmetry. On the other hand, it was shown that the one-loop and two-loop approximations, used as an input for the construction of "generators" $\beta(g)$ and $\gamma(g)$, yield expressions (20), (21) and (22) that obeys the group symmetry and exactly satisfy FEs (11) and (12).

More generally, one can state the following logical structure of the RGM procedure.

- Solving group equation(s) for invariant coupling(s) $\bar{g}_{\mathrm{rg}}(x, g)$ using some approximate solution $\bar{g}_{\mathrm{pt}}$ as an input.

- Obtaining RG solutions for some other QFT objects (like vertices and propagator amplitudes) on the basis of the expression(s) for $\bar{g}_{\mathrm{rg}}$ just derived. Typically, they satisfy the equation

$$
X M(x, y, g)=\gamma(y, g) M(x, y, g) .
$$

General structure of the corresponding solutions has the form

$$
M(x, y ; g)=z_{M}^{-1}(y, g) \mathcal{M}(x / y, \bar{g}(x, y ; g)) .
$$

Note that the function $\mathcal{M}$ in the r.h.s. depends only on the $\mathrm{RG}$ invariants, that is on the first integrals of the RG operator $X$ introduced in Eqs.(6) and (7). It satisfies homogeneous partial differential equations (PDEs) $X \mathcal{M}=0$. For the RG invariant objects, like observables, $z_{M}=1, \gamma=0$.

Now we can resume the RGM properties. The RGM is a regular procedure of combining dynamical information (taken from an approximate solution) with the RG symmetry. The essence of RGM is the following:

1) The mathematical tool used in RGM is Lie differential equations.

2) The key element of RGM is possibility of an (approximate) determination of "generators", like $\beta(g), \gamma(g)$, from dynamics.

3) The RGM works effectively in the case when the solution has a singular behaviour. It restores the structure of singularity compatible with the RG symmetry.

\section{Evolution of Renormalization Group}

In the 70s and 80s RG ideas have been applied to critical phenomena: spontaneous magnetization, polymerization, percolation, non-coherent radiation transfer, dynamic chaos, and so on. Less sophisticated motivation by Wilson in spin lattice phenomena (than in QFT) made this "explosion" of RG applications possible.

\section{$2.1 \quad$ Renormalization Group Evolving}




\subsubsection{Kadanoff-Wilson $R G$ in critical phenomena}

a. Spin lattice. The so-called renormalization group in critical phenomena is based on the Kadanoff-Wilson procedure [13, 14] referred to as "decimation" or "blocking". Initially, it emerged from the problem of spin lattice.

Imagine a regular (two- or three-dimensional) lattice consisting of $N^{d}, d=2,3$ cites with an 'elementary step' a between them. Suppose that at every site a spin vector $\boldsymbol{\sigma}$ is located. The Hamiltonian, describing the spin interaction between nearest neighbours

$$
H=k \sum_{i} \boldsymbol{\sigma}_{i} \cdot \boldsymbol{\sigma}_{i \pm 1}
$$

contains $k$, the coupling constant. A statistical sum is obtained from the partition function, $S=<\exp (-H / \theta)>$ aver .

To realize blocking, one has to perform the "spin averaging" over block consisting of $n^{d}$ elementary sites. This step diminishes the number of degree of freedom from $N^{d}$ to $(N / n)^{d}$. It also destroys the small-range properties of a system, in the averaging course some information being lost. However, the long-range physics (like correlation length essential for phase transition) is not affected by it, and we gain simplification of the problem.

As a result of this blocking procedure, new effective spins $\boldsymbol{\Sigma}$ arise in new sites forming a new effective lattice with a step na. We arrive also at the new effective Hamiltonian

$$
H_{\mathrm{eff}}=K_{n} \sum_{I} \Sigma_{I} \cdot \Sigma_{I \pm 1}+\Delta H
$$

with the effective coupling $K_{n}$ between new spins $\boldsymbol{\Sigma}_{I}$ of new neighbouring sites; $K_{n}$ has to be defined by the averaging process as a function of $k$ and $n$. Here, $\Delta H$ contains quartic and higher spin forms which are irrelevant for the IR (long-distance) properties. Due to this, one can drop $\Delta H$ and conclude that the spin averaging leads to an approximate transformation,

$$
k \sum_{i} \boldsymbol{\sigma} \cdot \boldsymbol{\sigma} \rightarrow K_{n} \sum_{I} \boldsymbol{\Sigma} \cdot \boldsymbol{\Sigma}
$$

or, taking into account the "elementary step" change, to $\left\{a \rightarrow n a, k \rightarrow K_{n}\right\}$. The latter is the Kadanoff-Wilson transformation. It is convenient to write down the new coupling $K_{n}$ in the form $K_{n}=K(1 / n, K)$. Then, the $\mathrm{KW}$ transformation reads

$$
K W_{n}:\left\{a \rightarrow n a, \quad k \rightarrow K_{n}=K(1 / n, k)\right\}
$$

These transformations obey composition law $K W_{n} \cdot K W_{m}=K W_{n m}$ if the relation

$$
K(x, k)=K(x / t, K(t, k)), \quad x=1 / n m, \quad t=1 / n .
$$

holds. This is very close to RG symmetry.

We observe the following points: 
- The RG symmetry is approximate (due to neglecting $\Delta H$ ).

- The transformations $K W_{n}$ are discrete.

- There exist no reverse transformation to $K W_{n}$.

- Transformations $K W_{n}$ relate different auxiliary models.

Hence, the "Kadanoff-Wilson renormalization group" (KW-RG) is an approximate and discrete semi-group. For a long-distance (IR limit) physics, however, $\Delta(1 / n)$ is small and it is possible to use differential Lie equations?

b. Polymer theory. In polymer physics, one considers statistical properties of polymer macromolecules which can be imagined as a very long chain of identical elements (with the number of elements $N$ as big as $10^{5}$ ). Molecules are swimming in a solvent and form globulars. This big molecular chain forms a specific pattern resembling the pattern of a random walk. The central problem of the polymer theory is very close to that of a random walk and can be formulated as follows.

For a long chain of $N$ "steps" (the size of step $=a$ ), one has to find the "chain size" $R_{N}$, the distance between the "start" and the "finish" points (the size a of globular), with the distribution function $f(\phi)$ of angles between the neighboring elements being given.

For large $N$ values, the molecular size $R_{N}$ follows the power Fleury law $R_{N} \sim N^{\nu}$ with $\nu$, the Fleury index. When $N$ is given, $R_{N}$ is a functional of $f(\phi)$ which depends on external conditions (e.g., temperature $T$, properties of solvent, etc. ). If $T$ grows, $R_{N}$ increases and at some moment globulars touch one another. This is the polymerization process which is very similar to a phase transition phenomenon.

The Kadanoff-Wilson blocking ideology has been introduced in physics of polymers by De Gennes [15. The key idea is a grouping of $n$ neigbouring elements of a chain into a new "elementary block". It leads to the transformation $\left\{1 \rightarrow n ; a \rightarrow A_{n}\right\}$ which is analogous to one for the spin lattice decimation. This transformation must be specified by a direct calculation which gives an explicit form of $A_{n}=\bar{a}(n, a)$. Here, we have a discrete semi-group. Then, by using the KW-RG technique, one finds the fixed point, obtains the Fleury power law and can calculate its index $\nu$.

The essential feature of a polymer chain is the impossibility of a self-intersection. This is known as an excluded volume effect in the random walk problem. Generally, the excluded volume effect yields some complications. However, inside the alternative, the QFT RG approach to polymers [16], it can be treated rather simply by introducing an one more argument which is similar to finite length $L$ in the transfer problem or particle mass $m$ in QFT.

Besides polymers, the KW-RG technique has been used in some fields of physics, like percolation, non-coherent radiation transfer [17], dynamical chaos [18] and some others.

\footnotetext{
${ }^{5}$ In application of these transformations to critical phenomena, the notion of a fixed point is important. Generally, a fixed point is associated with power-type asymptotic behavior. Note here that, contrary to the QFT case considered in Section 1.3.2, in phase transitions we deal with the IR stable point.
} 


\subsubsection{Bogoliubov symmetry outside QFT}

Meanwhile, the original QFT-RG approach proliferated into some other parts of theoretical physics. In the late 50s, it was used [19] for summation of Coulomb singularities in Bogoliubov's theory of superconductivity based on the Fröhlich electron-phonon interaction. Twenty years later it was used in the theory of turbulence.

a. Turbulence. To formulate the turbulence problem in terms of RG, one has to perform the following steps 20, 21]:

1. Introduce the generating functional for correlation functions.

2. Write down the path integral representation for this functional.

3. By changing the functional integration variable, find the equivalence of the statistical system to some quantum field theory model.

4. Construct the system of Schwinger-Dyson equations for this equivalent QFT model.

5. Perform the finite renormalization procedure and derive the RG equations.

Here, the reparameterization degree of freedom, physically corresponds to a change of long wave-length cutoff which is built-in into the definition of a few effective parameters.

b. Weak shock wave. Another example can be taken from hydrodynamics. Consider a weak shock wave in the one-dimensional case of a large distance $l$ from the starting (implosion) point. The dependence of velocity $v$ of a matter as a function of $l$ at a given moment of time $t$ has a simple triangular shape and can be described by the expression

$$
v(l)=\frac{l}{L} V \text { at } \quad l \leq L ; \quad=0 \text { for } \quad l>L,
$$

where $L=L(t)$ is the front position and $V=v(L)$ - the front velocity. They are functions of time. In the absence of viscosity, the "conservation law" $L V=$ Const. holds. Due to this, they can be treated as functions of the front wave position $L \equiv x, V=V(x)$ as well. If the physical situation is homogeneous, then the front velocity $V(x)$ should be considered as a function of only two additional relevant arguments - its own value $V_{0}=V\left(x_{0}\right)$ at some precedent point $\left(x_{0}<x\right)$ and of the $x_{0}$ coordinate. In can be written down in the form : $V(x)=G\left(x / x_{0}, V_{0}\right)$. If we pick up three points $x_{0}, x_{1}$ and $x_{2}$ (for details, see Refs. [22, 23]), then the initial condition may be given either at $x_{0}$ or $x_{1}$. Thus, we obtain the FE equation equivalent to (11)

$$
V_{2}=G\left(x_{2} / x_{0}, V_{0}\right)=G\left(x_{2} / x_{1}, V_{1}\right)=G\left(x_{2} / x_{1}, G\left(x_{1} / x_{0}, V_{0}\right)\right) .
$$

c. One-dimensional transfer. A similar argument has been done by Mnatzakanian [26] in the transfer problem at one dimension. Imagine a half-space filled with a homogeneous 
medium on the surface of which some flow (of radiation or particles) with intensity $g_{0}$ falls from the vacuum half-space.

Follow the flow as it moves inwards the medium at the distance $l$ from the boundary. Due to homogeneity along the $l$ coordinate, the intensity of the penetrated flow $g(l)$ depends on two essential arguments, $g(l)=G\left(l, g_{0}\right)$. The values of the flow at three different points $g_{0}$ (on the boundary), $g_{1}$ and $g_{2}>g_{1}$ can be connected with each other by the transitivity relations, $g_{1}=G\left(\lambda, g_{0}\right), \quad g_{2}=G\left(\lambda+l, g_{0}\right)=G\left(l, g_{1}\right)$, which lead to the FE

$$
G(l, g)=G(l-\lambda, G(\lambda, g)) .
$$

Performing a logarithmic change of variables $l=\ln x, \lambda=\ln t, G(l, g)=\bar{g}(x, g)$, we see that (27) is equivalent to (11).

Consider now intensity of a reverse flow, that is total amount of particles at the point $l$ moving in the backward direction. It is completely defined by $g_{0}$ and can be written down as $R\left(l, g_{0}\right)$. This function can be represented in the form $R(l, g)=R_{0}(g) N(l, g)$ with $R_{0} \equiv R(0, g)$ and function $N$ "normalized" on the boundary $N(0, g)=1$. Playing the same game with transitivity, we arrive at FE

$$
N(l, g)=Z(l, g) N\left(l-\lambda, G(l, G(\lambda, g)) ; \quad Z=R_{0}\left(g_{1}\right) / R_{0}(g)\right.
$$

related to Eq.(12) by logarithmic change of variables. One can refer to (27) and (28) as to the additive version of RG FEs and to previous equations of Section 1, like (11), (12) and (13) as to the multiplicative one.

The transfer problem admits a modification connected with discrete inhomogeneity: imagine the case of two different kinds of homogeneous materials separated by the inner boundary surface at $l=L$. The point of breaking $l=L$ may correspond to the boundary with empty space, and resulting equation is equivalent to Eq.(13).

One more generalization is related to "multiplication" of argument $g$ as expressed by Eq.(14). Physically, this relates to the case of radiation on different frequencies $\omega_{i}, i=$ $1,2, \ldots k$ (or particles of different energies or of different types).

Take the case of $k=2$ and suppose that the material of the medium has such properties that the transfer processes of the two flows are not independent. In this case, the characteristic functions of these flows $G$ and $H$ are dependent on both the boundary values $g_{0}$ and $h_{0}$ and can be taken as functions $g(l)=G\left(l, g_{0}, h_{0}\right), \quad h(l)=H\left(l, g_{0}, h_{0}\right)$.

After a group operation $l \rightarrow l-\lambda$, we arrive at a coupled set of functional equations $G(l+\lambda, g, h)=G\left(l, g_{\lambda}, h_{\lambda}\right), H(l+\lambda, g, h)=H\left(l, g_{\lambda}, h_{\lambda}\right) ; g_{\lambda} \equiv G(\lambda, g, h), h_{\lambda} \equiv H(\lambda, g, h)$ which is just an additive version of system (15) at $k=2$.

Now we can make the important conclusion that a common property yielding functional group equations is just the transitivity property of some physical quantity with respect to the way of giving its boundary or initial value.

Hence, the RG symmetry is not a symmetry of equations but a symmetry of solution, that is of equations and boundary conditions considered as a whole. 


\subsection{Difference between Bogoliubov RG and $\mathrm{KW}-\mathrm{RG}$}

As we have mentioned above, the RG ideas expanded in diverse fields of physics in two different ways:

- via direct analogy with the Kadanoff-Wilson construction (averaging over some set of degrees of freedom) in polymers, non-coherent transfer and percolation, i.e., constructing a set of models for a given physical problem.

- via finding an exact RG symmetry by proof of the equivalence with a QFT model (e.g., in turbulence [20, 21]), plasma turbulence [27]) or by some other reasoning (like in a transfer problem).

To the question Are there different renormalization groups? the answer is positive:

1. In QFT and some simple macroscopic examples, RG symmetry is an exact symmetry of the solution formulated in its natural variables.

2. In turbulence, continuous spin-field models and some others, it is a symmetry of an equivalent QFT model.

3. In polymers, percolation, etc. , (with $\mathrm{KW}$ blocking), the $\mathrm{RG}$ transformation is a transformation between different auxiliary models (specially constructed for this purpose) of a given system.

As we have shown, there is no essential difference in the mathematical formulation. There exists, however, a profound difference in physics:

- In the cases 1 and 2 (as well as in some macroscopic examples), the RG is an exact symmetry of a solution.

- In the Kadanoff-Wilson type problem (spin lattice, polymers, etc. ), one has to construct a set $\mathcal{M}$ of models $M_{i}$. The KW-RG transformation

$$
K W_{n} M_{i}=M_{n i}, \text { with integer } n
$$

is acting inside a set of models.

\subsection{Functional self-similarity}

The RG transformations have close connection with the concept of self-similarity. The self-similarity transformations for problems formulated by nonlinear PDEs are well known since the last century, mainly in dynamics of liquids and gases. They are one parameter $\lambda$ transformations defined as a simultaneous power scaling of independent variables $z=$ $\{x, t, \ldots\}$, solutions $f_{k}(z)$ and other functions $V_{i}(z)$ (like external force)

$$
S_{\lambda}:\left\{x^{\prime}=x \lambda, t^{\prime}=t \lambda^{a}, f_{k}^{\prime}=\lambda^{\varphi_{k}} f_{k}, V_{i}^{\prime}=\lambda^{\nu_{i}} V_{i}\right\}
$$


entering into the equations.

To emphasize their power structure, we use a term power self-similarity $=$ PS. According to Zel'dovich and Barenblatt, [28] the PS can be classified as:

a/ $P S$ of the 1 st kind with all indices $a, \ldots \varphi, \nu, \ldots$ being integers or rational (Rational PS) that are usually found from the theory of dimensions;

b/ PS of the 2nd kind with irrational indices (Fractal PS) which should be defined from dynamics.

To relate RG with PS, turn to the renormgroup FE $\bar{g}(x t, g)=\bar{g}(x, \bar{g}(t, g))$. Its general solution is known; it depends on an arbitrary function of one argument - see Eq.(19). However, at the moment, we are interested in a special solution linear in the second argument: $\bar{g}(x, g)=g X(x)$. The function $X(x)$ should satisfy the equation $X(x t)=$ $X(x) X(t)$ with the solution $X(x)=x^{\nu}$. Hence, $\bar{g}(x, t)=g x^{\nu}$. This means that in our special case, linear in $g$, the RG transformation (10) is reduced to the PS transformation,

$$
R_{t} \quad \Rightarrow \quad S_{t}:\left\{x^{\prime}=x t^{-1}, g^{\prime}=g t^{\nu}\right\}
$$

Generally, in RG, instead of a power law, we have an arbitrary functional dependence. Thus, one can consider transformations (10), (13) and (16) as functional generalizations of usual (i.e., power) self-similarity transformations. Hence, it is natural to refer to them as to the transformations of functional scaling or functional (self)similarity (FS) rather than to RG-transformations. In short,

$$
\mathrm{RG} \equiv \mathrm{FS}
$$

with FS standing for Functional Similarity"

Now we can answer the question on the physical meaning of the symmetry underlying FS and the Bogoliubov renormgroup. As we have mentioned, it is not a symmetry of a physical system or of equation(s) of the problem at hand, but a symmetry of a solution considered as a function of the relevant physical variables and suitable boundary parameters. A symmetry like that can be related, in particular, to the invariance of a physical quantity described by this solution with respect to the way in which the boundary conditions are imposed. The changing of this way constitutes a group operation in the sense that the group composition law is related to the transitivity property of such changes.

Homogeneity is an important feature of a physical system under consideration. However, homogeneity can be violated in a discrete manner. Imagine that such a discrete violation is connected with a certain value of $x$, say, $x=y$. Here, RG transformation with the canonical parameter $t$ has the form (13).

The symmetry connected with FS is a very simple and frequently encountered property of physical solutions. It can easily be "discovered" in numerous problems of theoretical physics like classical mechanics, transfer theory, classical hydrodynamics, and so on [30, 26, 22, 23] - see, above, Section 2.1.2.

\footnotetext{
${ }^{6}$ This notion was first mentioned in [29] and formally introduced [30] in the beginning of 80s.
} 


\section{Symmetry of solution in Mathematical Physics}

\subsection{Constructing RG-symmetries and their use}

From the discussion in Sections 1.1 and 1.2 it follows that FS transformation in QFT is the scaling transformation of an independent variable $x$ (and, possibly, the parameter $y$ ) accompanied by a functional transformation of the solution characteristic $g$. It is introduced by means of either finite transformations (10), (13) and (16) or the infinitesimal operator (7). Hence, the symmetry of a solution, i.e., FS symmetry, is commonly understood in QFT as the Lie point symmetry of a one-parameter transformation group defined by the operator of the (7)-type.

Now, we are interested in getting answers to the following questions:

- is it possible to extend the notion of RG symmetry (RGS) and generalize the form of RGS implementation that may differ from that given by (17) ? - and if "yes",

- is it possible to create a regular algorithm of finding these symmetries ?

The answer is positive to both the questions, and below we demonstrate the regular algorithm of constructing RGS in mathematical physics that up to now has been devised only for boundary value problem (BVP) for the (system of) differential equation(s) which we shall refer to as basic equations (BEs). The point is that these models can be analyzed by methods of Lie group analysis which employ infinitesimal group transformations instead of the finite one.

The general idea of the algorithm is to find a specific renormgroup manifold $\mathcal{R} \mathcal{M}$ that contains the desired solution of BVP. Then construction of a RGS, that leaves this solution unaltered, is performed by standard methods of a group analysis of DEs.

The regular algorithm of constructing RGS (and their application) can be formulated in a form of a scheme which comprises a few steps. It is illustrated in Figure 1.

I. First of all, a specific renormgroup manifold $\mathcal{R} \mathcal{M}$ for the given BVP should be constructed which is identified below with a system of the $k$ th-order DEs

$$
F_{\sigma}\left(z, u, u_{(1)}, \ldots, u_{(k)}\right)=0, \quad \sigma=1, \ldots, s .
$$

In (31) and what follows we use terminology of group analysis and the notation of differential algebra. In contrast with the mathematical analysis, where we usually deal with functions $u^{\alpha}, \alpha=1, \ldots, m$ of independent variables $x^{i}, i=1, \ldots, n$ and derivatives $u_{i}^{\alpha}(x) \equiv \partial u^{\alpha} / \partial x^{i}, u_{i j}^{\alpha}(x) \equiv \partial^{2} u^{\alpha} / \partial x^{i} \partial x^{j}, \ldots$ that are also considered as functions of $x$, in differential algebra we treat $u^{\alpha}, u_{i}^{\alpha}, u_{i j}^{\alpha}, \ldots$ as variables as well. Therefore, in differential algebra we deal with an infinite number of variables

$$
x=\left\{x^{i}\right\}, \quad u=\left\{u^{\alpha}\right\}, \quad u_{(1)}=\left\{u_{i}^{\alpha}\right\}, \quad u_{(2)}=\left\{u_{i_{1} i_{2}}^{\alpha}\right\}, \ldots ; \quad\left(i, i_{1}, \ldots=1, \ldots, n\right),
$$

\footnotetext{
${ }^{7}$ In the present form this scheme was described in [31]. One can find there historical comments and references on the pioneering publications.
} 


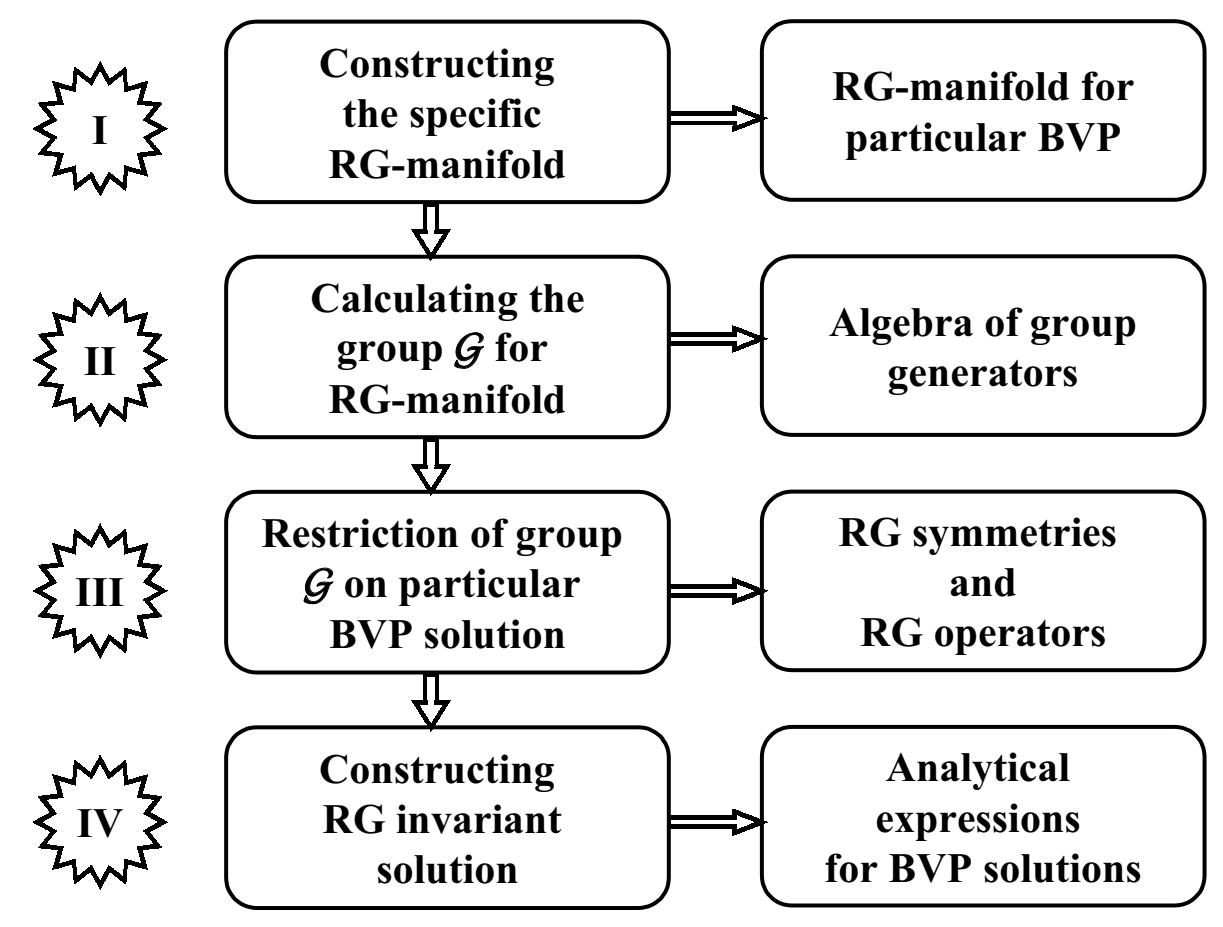

Figure 1: RGS constructing and application to BVP in Mathematical Physics.

where $x^{i}$ are called independent variables, $u^{\alpha}$ dependent variables and $u_{(1)}, u_{(2)}, \ldots$ derivatives. A locally analytic function $f\left(x, u, u_{(1)}, \ldots, u_{(k)}\right)$ of variables (32), with the highest $k$ th-order derivative involved, is called a differential function of order $k$. The set of all differential functions of a given order form a space of differential functions $\mathcal{A}$, the universal space of modern group analysis [32] - 35].

A particular way of realization of the first step can hardly be described uniquely, as it depends on both a form of basic equations and a boundary condition; generally, $\mathcal{R} \mathcal{M}$ does not coincide with BEs. We indicate here a few feasible routines for this step.

- One can use an extension of the space of variables involved in group transformations. These variables, e.g., may be parameters, $p=\left\{p^{j}\right\}, j=1, \ldots, l$ entering into a solution via the equations and/or boundary conditions. Adding parameters $p$ to the list of independent variables $z=\{x, p\}$ we treat BEs in this extended space as $\mathcal{R} \mathcal{M}$ (31). Similarly, one can extend the space of differential variables by treating derivatives with respect to $p$ as additional differential variables.

- Another possibility employs reformulating of boundary conditions in terms of embedding equations or differential constraints which are then combined with BEs. The key idea here is to treat the solution of BVP as an analytic function of independent variables and boundary parameters $b=\left\{x_{0}^{i}, u_{0}^{\alpha}\right\}$ as well. Differentiation with re- 
spect to these parameters gives additional DEs (embedding equations) that, together with BEs, form $\mathcal{R} \mathcal{M}$. In some cases, while calculating Lie point RGS, the role of embedding equations can be played by differential constraints (for details see [31]) that come from an invariance condition for BEs with respect to the Lie-Bäcklund symmetry group.

- In the case when BEs contain a small parameter $\alpha$, the desired $\mathcal{R} \mathcal{M}$ can be obtained by simplification of these equations and use of "perturbation methods of group analysis" (see Vol.3, Chapter 2, p.31 in [34]). The main idea here is to consider a simplified $(\alpha=0)$ model, which admits a wider symmetry group (see examples in the Section 4.2 below) in comparison with the case $\alpha \neq 0$. When we take the contributions from small $\alpha$ into account, this symmetry is inherited by BEs, which results in the additional terms, corrections in powers of $\alpha$, in the RGS generator.

II. The next step consists in calculating the most general symmetry group $\mathcal{G}$ that leaves the manifold $\mathcal{R} \mathcal{M}$ unaltered. The term "symmetry group", as used in the classical group analysis, means the property of the system (31) to admit a local Lie group of point transformations in the space $\mathcal{A}$.

The Lie algorithm of finding such symmetries consists in constructing tangent vector fields defined by the operator

$$
X=\xi^{i} \partial_{x^{i}}+\eta^{\alpha} \partial_{u^{\alpha}}, \quad \xi^{i}, \eta^{\alpha} \in \mathcal{A},
$$

with the coordinates, $\xi^{i}, \eta^{\alpha}$ that are functions of group variables and have to be determined by a system of equations

$$
X F_{\sigma \mid(31)}=0, \quad \sigma=1, \ldots, s
$$

that follow from the invariance of $\mathcal{R} \mathcal{M}$. Here $X$ is extended $[$ to all derivatives involved in $F_{\sigma}$ and the symbol $\mid 31$ means calculated on the frame (31). A system of linear homogeneous PDEs (34) for coordinates $\xi^{i}, \eta^{\alpha}$, known as determining equations, is an overdetermined system as a rule. The solution of Eqs.(34) define a set of infinitesimal operators (33) (also known as group generators), which correspond to the admitted vector field and form a Lie algebra. In the case that the general element of this algebra

$$
X=\sum_{j} A^{j} X_{j}
$$

where $A^{j}$ are arbitrary constants, contains finite number of operators, $1 \leq j \leq l$, the group is called finite-dimensional (or simply finite) with the dimension $l$; otherwise, for

\footnotetext{
${ }^{8}$ We use here the terminology adopted in Russian literature [32, 35]. This symmetry is also known as generalized or higher-order symmetry [33, 34].

${ }^{9}$ The extending of generators to the derivatives employs the prolongation formulas and is a regular procedure in group analysis (see, e.g. [34]).
} 
unlimited $j$ or in the case that coordinates $\xi^{i}, \eta^{\alpha}$ depend upon arbitrary functions of group variables, the group is called infinite.

The use of the infinitesimal criterion (34) for calculating the symmetry groups makes the whole procedure algorithmic and can be carried out not only "by hand" but using the symbolic packages of the computer algebra (see, e.g., Vol.3 in [34]) as well. In modern group analysis, different modifications of the classical Lie scheme are in use (see [32, 33, 34] and references therein).

The generator (33) of the group $\mathcal{G}$ is equivalent to the canonical Lie-Bäcklund operator

$$
Y=\kappa^{\alpha} \partial_{u^{\alpha}}, \quad \kappa^{\alpha} \equiv \eta^{\alpha}-\xi^{i} u_{i}^{\alpha},
$$

that is known as a canonical representation of $X$ and plays an essential role in RGS constructing.

However, the group defined by the generators (33) and (36) cannot yet be referred to as a renormgroup, as it is not related to a partial BVP solution of interest.

III. To obtain RGS, the restriction of the group $\mathcal{G}$ on particular BVP solution should be made which forms the third step. Mathematically, this procedure appears as checking the vanishing condition for the linear combination of coordinates $\kappa_{j}^{\alpha}$ of the canonical operator equivalent to (35) on a particular approximate (or exact) BVP solution $U^{\alpha}(z)$

$$
\left\{\sum_{j} A^{j} \kappa_{j}^{\alpha} \equiv \sum_{j} A^{j}\left(\eta_{j}^{\alpha}-\xi_{j}^{i} u_{i}^{\alpha}\right)\right\}_{u^{\alpha}=U^{\alpha}(z)}=0 .
$$

Evaluating (B7) on a particular BVP solution $U^{\alpha}(z)$ transforms the system of DEs for group invariants into algebraic relations $\mathrm{\Phi D}$. Firstly, it gives relations between $A^{j}$ thus "combining" different coordinates of group generators $X_{j}$ admitted by the $\mathcal{R} \mathcal{M}$ (31). Secondly, it eliminates (partially or entirely) the arbitrariness that may appear in coordinates $\xi^{i}, \eta^{\alpha}$ in the case of an infinite group $\mathcal{G}$. In terms of the "classic" QFT RG terminology, where it exists only one operator $X$ of (17)-type (i.e., all $A_{j}$ except one are equal to zero), the procedure of group restriction on a particular BVP solution $\bar{g}_{a p p r}$ eliminates arbitrariness in the form of $\beta(g)$-function.

While the general form of the condition given by Eq.(37) is the same for any BVP solution, the way of realization of the restriction procedure in every particular case employs a particular perturbation approximation (PA) for the concrete BVP.

Generally, the restriction procedure reduces the dimension of $\mathcal{G}$. It also "fits" boundary conditions into the operator (35) by a special choice of coefficients $A_{j}$ and/or by choosing the particular form of arbitrary functions in coordinates $\xi^{i}, \eta^{\alpha}$. Hence, the general element (35) of the group $\mathcal{G}$ after the fulfillment of a restriction procedure is expressed as a linear

\footnotetext{
${ }^{10}$ Similar relations were discussed in [32], Chapter 8, when constructing invariant solutions for the Cauchy problem for a quasi-linear system of first order PDEs.
} 
combination of new generators $R_{i}$ with the coordinates $\tilde{\xi}^{i}, \tilde{\eta}^{\alpha}$,

$$
X \Rightarrow R=\sum_{j} B^{j} R_{j}, \quad R_{j}=\tilde{\xi}_{j}^{i} \partial_{x^{i}}+\tilde{\eta}_{j}^{\alpha} \partial_{u^{\alpha}}
$$

where $B^{j}$ are arbitrary constants.

The set of RGS generators $R_{i}$ each containing the desired BVP solution in its invariant manifold, define a group of transformations that we also refer to as renormgroup. Therefore, here we extend the notion of renormgroup and RG symmetry and the direct analogy with the "Bogoliubov RG" is preserved only for one-parameter group of point transformations.

IV. The prescribed three steps entirely define the regular algorithm of RGS construction but do not touch on how a BVP solution is found. Hence, one more important, the fourth, step should be added. It consists in using RGS generators to find analytical expressions for the new, "improved", solution of the BVP.

Mathematically, this step makes use of $\mathrm{RG}=\mathrm{FS}$ invariance conditions that are given by a combined system of (31) and the vanishing condition for the linear combination of coordinates $\tilde{\kappa}_{j}^{\alpha}$ of the canonical operator equivalent to (38),

$$
\sum_{j} R^{j} \tilde{\kappa}_{j}^{\alpha} \equiv \sum_{j} B^{j}\left(\tilde{\eta}_{j}^{\alpha}-\tilde{\xi}_{j}^{i} u_{i}^{\alpha}\right)=0
$$

One can see that conditions (39) are akin to (37). However, in contrast with the previous step, the differential variables $u$ in (39) should not be replaced by an approximate expression for the BVP solution $U(z)$, but should be treated as usual dependent variables.

For the one-parameter Lie point renormgroup, RG invariance conditions lead to the first order $P D E$ that gives rise to the so-called group invariants (like invariant couplings in QFT) which arise as solutions of associated characteristic equations.

A general solution of the BVP is now expressed in terms of these invariants. On the one hand, this is in direct analogy with the structure of RG invariant solutions in QFT compare with Eqs.(22) and (24).

On the other hand, it reminds the so-called $\Pi$-theorem from the theory of dimensional analysis and similitude (see, Section 19 in [32], Section 6 of Chapter 1 in [36] and historical comment to Section 43 in [37]) directly related to power self-similarity, discussed above in Section 2.3.

However, as we shall see later, in the general case of arbitrary RGS the group invariance condition obtained for BVP is not necessarily characteristic equations for the Lie point group operator. They may appear in a more complicated form, e.g., as a combination of PDE and higher order ODE (see Section 4.2). Nevertheless, the general idea of finding solution of the BVP as RG invariant solutions remains valid. 


\subsection{Examples of solution improving}

We present now a few examples of the RGS construction with further use of the symmetry for "improving" an approximate solution.

\subsubsection{Modified Burgers equation}

As the first example, we take the initial value problem for the modified Burgers equation

$$
u_{t}-a u_{x}^{2}-\nu u_{x x}=0, \quad u(0, x)=f(x) .
$$

It is connected with the heat equation

$$
\tilde{u}_{t}=\nu \tilde{u}_{x x}
$$

by transformation $\tilde{u}=\exp (a u / \nu)$ and has an exact solution. Due to this, while using RGS to find a solution, one can check the validity of our approach. The RGS constructing for (40) is an apt illustration of the general scheme, shown in Figure 1 which may be helpful in understanding other examples of the general algorithm implementation. We review here in short the procedure and results of paper Ref. [38].

The RG-manifold $\mathcal{R} \mathcal{M}$ (step I) is given by Eq.(40) with the parameters of nonlinearity $a$ and dissipation $\nu$ included in the list of independent variables. The Lie calculational algorithm applied to $\mathcal{R} \mathcal{M}$ gives, for the admitted group $\mathcal{G}$ (step II), nine independent terms in the general expression for the group generator

$$
\begin{gathered}
X=\sum_{i=1}^{8} A^{i}(a, \nu) X_{i}+\alpha(t, x, a, \nu) e^{-a u / \nu} \partial_{u}, \\
X_{1}=4 \nu t^{2} \partial_{t}+4 \nu t x \partial_{x}-(\nu / a)\left(x^{2}+2 \nu t\right) \partial_{u}, \quad X_{2}=2 t \partial_{t}+x \partial_{x}, \\
X_{3}=(1 / \nu) \partial_{t}, \quad X_{4}=2 \nu t \partial_{x}-(\nu / a) x \partial_{u}, \quad X_{5}=\partial_{x}, \quad X_{6}=-(\nu / a) \partial_{u}, \\
X_{7}=a \partial_{a}+[(\nu / a)-u] \partial_{u}, \quad X_{8}=2 \nu \partial_{\nu}+x \partial_{x}+2[u-(\nu / a)] \partial_{u} .
\end{gathered}
$$

Here, $A^{i}(a, \nu)$ are arbitrary functions of their arguments and $\alpha(t, x, a, \nu)$ is an arbitrary function of four variables, satisfying the heat equation (41).

A set of operators $X_{i}$ form an eight-dimensional Lie algebra $L_{8}$. The first six generators relate to the well-known symmetries of the modified (potential) Burgers equation (see, e.g., Vol.1, p.183 in [34]). They describe projective transformation in the $(t, x)$-plane $\left(X_{1}\right)$, dilatations in the same plane $\left(X_{2}\right)$, translations along the $t-, x-$ and $u$-axes $\left(X_{3}\right.$, $X_{5}$ and $\left.X_{6}\right)$ and Galilean transformations $\left(X_{4}\right)$. The last two generators $X_{7}$ and $X_{8}$ relate to dilatations of parameters $a$ and $\nu$ now involved in group transformations. 
The procedure of restriction (step III) of the group (42) admitted by $\mathcal{R} \mathcal{M}$ (40) implies the check of the invariance condition (37) on a particular BVP solution $u=U(t, x, a, \nu)$

$$
\left\{\eta_{\infty}+\sum_{i=1}^{8} A^{i}(a, \nu) \kappa_{i}\right\}_{\mid u=U(t, x, a, \nu)}=0, \quad \kappa_{i} \equiv \eta_{i}-\xi_{i}^{1} u_{t}-\xi_{i}^{2} u_{x}-\xi_{i}^{3} u_{a}-\xi_{i}^{4} u_{\nu}
$$

This formula expresses the coordinate $\alpha$ of the last term in (42) via the remaining coordinates of eight generators $X_{i}$ for arbitrary $t$, and hence for $t=0$, when $U(0, x, a, \nu)=f(x)$. As a result, we obtain the "initial" value $\alpha(0, x, a, \nu)$ and then, using the standard representation for the solution to the linear parabolic equation (41), the value of $\alpha$ at arbitrary $t \neq 0$

$$
\alpha(t, x, a, \nu)=-\sum_{i=1}^{8} A^{i}(a, \nu)<\bar{\kappa}_{i}(x, a, \nu)>
$$

Here, $\bar{\kappa}_{i}(x, a, \nu)$ denote "partial" canonical coordinates $\kappa_{i}$ taken at $t=0$ and $u=f(x)$. Symbol $\langle F\rangle$ designates the convolution of a function $F$ with the fundamental solution of (41), multiplied by the exponential function of $f$ entering into the boundary condition

$$
<F(x, t, a, \nu)>\equiv \frac{1}{\sqrt{4 \pi \nu t}} \int_{-\infty}^{\infty} d y F(y, t, a, \nu) \exp \left(-\frac{(x-y)^{2}}{4 \nu t}+\frac{a f(y)}{\nu}\right) .
$$

Substitution (44) in the general expression (42) gives the desired RG generators

$$
\begin{gathered}
R_{i}=X_{i}+\varrho_{i} e^{-a u / \nu} \partial_{u}, \\
\varrho_{1}=\frac{\nu}{a}<x^{2}>, \quad \varrho_{2}=<x f_{x}>, \quad \varrho_{3}=\frac{1}{\nu}<a f_{x}^{2}+\nu f_{x x}>, \quad \varrho_{4}=\frac{\nu}{a}<x>, \\
\varrho_{5}=<f_{x}>, \quad \varrho_{6}=\frac{\nu}{a}<1>, \quad \varrho_{7}=<f-\frac{\nu}{a}>, \quad \varrho_{8}=<x f_{x}-2 f+2 \frac{\nu}{a}>.
\end{gathered}
$$

Operators $R_{i}$ form an eight-dimensional $R G$ algebra $R L_{8}$ that has the same tensor of structural constants as $L_{8}$, i.e. $R L_{8}$ and $L_{8}$ are isomorphic. Hence, the procedure of the group restriction eliminates the arbitrariness presented by the function $\alpha$ and "fits" the boundary conditions into RG generators by means of $\varrho_{i}$.

It can be verified that the exact solution of the initial-value problem (40)

$$
u(t, x ; a, \nu)=\frac{\nu}{a} \ln <1>\equiv \frac{\nu}{a} \ln \left\{\frac{1}{\sqrt{4 \pi \nu t}} \int_{-\infty}^{\infty} d y \exp \left(-\frac{(x-y)^{2}}{4 \nu t}+\frac{a f(y)}{\nu}\right)\right\}
$$

is the invariant manifold for any of the above RGS operators. And vice versa, (45) can be reconstructed from an approximate solution with the help of any of the RGS operators or their linear combination. For example, two such operators, $\nu R_{3} \equiv R_{t}$ and $(1 / a)\left(R_{6}+R_{7}\right) \equiv R_{a}$ were used in [38] to reconstruct the exact solution from perturbative 
(in time and in nonlinearity parameter $a$ ) solutions. Below, we describe this procedure (step IV) using the operator $R_{a}$,

$$
R_{a}=\partial_{a}+(1 / a)\left(-u+e^{-a u / \nu}<f(x)>\right) \partial_{u} .
$$

It is evident that $t, x$ and $\nu$ are invariants of group transformations with (46), whilst finite $\mathrm{RG}$ transformations of the two remaining variables, $a$ and $u$, are obtained by solving the Lie equations for (46), with $\ell$ the group parameter

$$
\frac{d a^{\prime}}{d \ell}=1,\left.\quad a^{\prime}\right|_{\ell=0}=a ; \quad \frac{d u^{\prime}}{d \ell}=\alpha\left(t, x, a^{\prime}, \nu\right) e^{-a^{\prime} u^{\prime} / \nu}-\frac{u^{\prime}}{a^{\prime}},\left.\quad u^{\prime}\right|_{\ell=0}=u .
$$

Combining these equations yields one more invariant $\mathcal{J}=e^{a u / \nu}-<1>$ for the RGS generator (46). Solution of (47) along with (44) gives the formulae for finite RG transformations of the group variables $\{t, x, a, \nu, u\}$

$$
t^{\prime}=t, x^{\prime}=x, \nu^{\prime}=\nu, a^{\prime}=a+\ell, u^{\prime}=\frac{\nu}{a+\ell} \ln \left(e^{a u / \nu}+<e^{\ell f(x) / \nu}-1>\right) .
$$

Choosing the value $a$ equal to zero, which is a starting point of PA in $a$, we get $a^{\prime}=\ell$. Then after excluding $t, x, \nu$ and $\ell$ from the expression for $u^{\prime}$ (48) and omitting accents over $t^{\prime}, x^{\prime}, \nu^{\prime}, u^{\prime}$ and $a^{\prime}$ the desired BVP solution (45) is obtained. It also follows directly from $\mathcal{J}$ in view of the initial condition $\left.\mathcal{J}\right|_{a=0}=0$.

A similar procedure can be fulfilled for the other RG operator,

$$
R_{t}=\partial_{t}+e^{-a u / \nu}<a f_{x}^{2}+\nu f_{x x}>\partial_{u}
$$

which is consistent with the PA in time $t$. Although invariants for $R_{t}$ and finite RG transformations differ from that for (46), the final result, i.e., the exact solution of BVP (40) given by (45), is the same. This possibility is the distinct demonstration of the multi-dimensional RGS to reconstruct the unique BVP solution from different PA: either in parameter $a$ or in $t$ (though we used only two one-dimensional subalgebras here

\subsubsection{BVP for ODE: simple example}

Quite recently, the QFT renormalization group ideology has been applied, a bit straightforward, in mathematical physics for asymptotic analysis of solutions to DEs [41, 42] and in constructing an envelope of the family of solutions [43].

Our second methodological example with linear ODE is presented here in order to illustrate the difference between our approach and the "perturbative RG theory" devised in 41] for a global analysis of BVP solutions in mathematical physics.

Consider a linear second order ODE for $y(t)$ with the initial conditions at $t=\tau$,

$$
y_{t t}+y_{t}+\varepsilon y=0, \quad y(\tau)=\tilde{u}, \quad y_{t}(\tau)=\tilde{w},
$$

\footnotetext{
${ }^{11}$ This can be considered as a construction parallel to the one used in Ref.[39].
} 
which has the exact solution:

$$
y=C_{+} e^{-\gamma_{+}(t-\tau)}+C_{-} e^{-\gamma_{-}(t-\tau)}, \gamma_{ \pm}=\frac{1 \pm K}{2}, K=\sqrt{1-4 \varepsilon}, C_{ \pm}=\mp \frac{\tilde{w}+\gamma_{\mp} \tilde{u}}{K} .
$$

Provided that the parameter $\varepsilon$ is small, the solutions to Eq.(49) has been treated in [41] with the aim to demonstrate effectiveness of the "perturbative RG theory" for an asymptotic analysis of a solution behaviour. The main goal of this treatment was to improve a perturbative expansion in powers of $\varepsilon$ with secular terms $\propto \varepsilon(t-\tau)$ and obtain $[2$ a uniformly valid asymptotic of a solution

$$
\begin{gathered}
y=c_{+} e^{(-1+\varepsilon(1+\varepsilon))(t-\tau)}+c_{-} e^{-\varepsilon(1+\varepsilon)(t-\tau)}+O\left(\varepsilon^{2}\right), \\
c_{+} \approx-((1+2 \varepsilon) \tilde{w}+\varepsilon \tilde{u}), \quad c_{-} \approx((1+2 \varepsilon) \tilde{w}+(1+\varepsilon) \tilde{u}),
\end{gathered}
$$

which is accurate for small values $\varepsilon \ll 1$ but for arbitrary values of the product $\varepsilon(t-\tau)$.

We are going to show that the use of our regular RG algorithm enables one to improve a PA solution (either in powers of $\varepsilon$ or in $t-\tau$ ) up to the exact BVP solution (50).

Rewriting (49) in the form of the system of two first order ODEs for functions $u \equiv y$ and $w \equiv y_{t}$,

$$
u_{t}=w, \quad w_{t}=-\varepsilon w-u,
$$

we construct $\mathcal{R} \mathcal{M}$ (step I) using the invariant embedding method (this approach has first been realized in [44]). Then, $\mathcal{R} \mathcal{M}$ is presented as a joint system of BEs (52) and embedding equations

$$
u_{\tau}-(\varepsilon \tilde{w}+\tilde{u}) u_{\tilde{w}}-\tilde{w} u_{\tilde{u}}=0, \quad w_{\tau}-(\varepsilon \tilde{w}+\tilde{u}) w_{\tilde{w}}-\tilde{w} w_{\tilde{u}}=0,
$$

treated in the extended space of group variables which include the parameters $\tau, \tilde{w}, \tilde{u}$ of boundary conditions in addition to $t$ and dependent variables $u, w$.

Omitting tedious calculations related to the following two steps (steps II and III), we present here two examples of resulting RGS generators

$$
\begin{aligned}
R_{\tau}=\partial_{\tau} & -(\tilde{w}+\varepsilon \tilde{u}) \partial_{\tilde{w}}+\tilde{w} \partial_{\tilde{u}}, \\
R_{\varepsilon}=\partial_{\varepsilon} & -\left(\frac{1}{\mu^{2}}(2 w+u)(1-t / 2)+(t / 2) u\right) \partial_{w}+\frac{t}{\mu^{2}}(2 w+u) \partial_{u} \\
& -\left(\frac{1}{\mu^{2}}(2 \tilde{w}+\tilde{u})(1-\tau / 2)+(\tau / 2) \tilde{u}\right) \partial_{\tilde{w}}+\frac{\tau}{\mu^{2}}(2 \tilde{w}+\tilde{u}) \partial_{\tilde{u}},
\end{aligned}
$$

12 The algorithm used in 41 for improving PA solutions with secular terms involves $a$ ) an introduction of some additional parameters in solutions, b) a special choice of these parameters that eliminates secular divergencies, and c) imposing a condition of independence of a solution upon the way of introducing these parameters. In some cases, this algorithm, directly borrowed from QFT RG-method, gives an exact solution. However, the question of correspondence of this construction to a transformation group of a solution of BEs remains open. 
that involve the initial values $\tilde{w}, \tilde{u}$ and initial point $\tau$ in RG transformations. In addition, $R_{\varepsilon}$ transforms the parameter $\varepsilon$.

Now, the procedure of constructing the BVP solution (50) (step IV) is similar to that used in the previous Section 3.2.1 and employ finite transformations that are defined by the Lie equations for the operators (53). For $R_{\tau}$ functions $u, w$ and the parameter $\varepsilon$ are group invariants, while the translations of $\tau$ and the corresponding transformations of $\tilde{u}, \tilde{w}$ restores the exact solution (50) from the PA in powers of $t-\tau$ (note that the parameter $\varepsilon$ is not necessarily small in this PA!).

For $R_{\varepsilon}$ the difference $t-\tau$ is group invariant, whilst the transformation of $\varepsilon$ and related transformations of $u, w, \tilde{u}, \tilde{w}$ restore the exact solution (50) from the PA (discussed in 41]) in powers of $\varepsilon$. Hence, as in the previous Section 3.2.1, both the RGS generators (53) reconstruct the unique BVP solution from different PAs.

\section{$4 \quad$ RG in Nonlinear optics}

\subsection{Formulation of a problem}

As a problem of real physical interest, take BVP that describes self-focusing of a highpower light beam. While the problem plays an important role in nonlinear electrodynamics since 60s, the detailed quantitative understanding of self-focusing is still missing [45, and there is no method which allows to find an analytic solution to the corresponding equations with arbitrary boundary conditions.

Here, we demonstrate the great potential of the RGS approach in constructing analytic solutions of BVP equations with arbitrary boundary conditions. The RGS method allows to consider different types of BEs for self-focusing process which include plane and cylindrical beam geometry, nonlinear refraction and diffraction. The merit of the RGS method is that it describes BVP solutions with one- or two-dimensional singularities in the entire range of variables from the boundary up to the singularity point.

Let us start with BVP for the system of two DEs

$$
\begin{gathered}
v_{z}+v v_{x}-\alpha n_{x}=0, \quad n_{z}+n v_{x}+v n_{x}+(\nu-1)(n v / x)=0, \\
v(0, x)=0, \quad n(0, x)=N(x),
\end{gathered}
$$

which are used in nonlinear optics of self-focusing wave beams when diffraction is negligible.

We study spatial evolution of the derivative of the beam eikonal $v$ and the beam intensity $n$ in the direction inwards the medium $z$ and in the transverse direction $x$. The term proportional to $\alpha$ is related to nonlinear refraction effects; $\nu=1$ and $\nu=2$ refers to the plane and cylindrical beam geometry, respectively. Boundary conditions (55) correspond to the plane front of the beam and the arbitrary transverse intensity distribution. 


\subsection{Plane geometry}

In the plane beam geometry (at $\nu=1$ ) Eqs. (54) can be reduced to the system of BEs

$$
\tau_{w}-n \chi_{n}=0, \quad \chi_{w}+\alpha \tau_{n}=0
$$

for functions $\tau=n z$ and $\chi=x-v z$ of $w=v / \alpha$ and $n$ arguments, with boundary conditions

$$
\tau(0, n)=0, \quad \chi(0, n)=H(n),
$$

where $H(n)$ is the inverse to $N(x)$. Here, the procedure of RGS constructing makes use of the Lie-Bäcklund symmetry and is described as follows [31]. The manifold $\mathcal{R} \mathcal{M}$ (step I) is defined by Eqs. (56) treated in the extended space that include dependent and independent variables $\tau, \chi, w, n$ and derivatives of $\tau$ and $\chi$ with respect to $n$ of an arbitrary high order. The admitted symmetry group $\mathcal{G}$ (step II) is represented by the canonical Lie-Bäcklund operator

$$
X=f \partial_{\tau}+g \partial_{\chi}
$$

with the coordinates $f$ and $g$ that are linear combinations of $\tau$ and $\chi$ and their derivatives $\partial^{i} \tau / \partial n^{i}$ and $\partial^{i} \chi / \partial n^{i}, i \geq 1$ with the coefficients depending on $w$ and $n$.

The restriction of the group admitted by $\mathcal{R} \mathcal{M}$ (56) (step III)) implies the check of the invariance condition (37) that yields two relations

$$
f=0, \quad g=0 .
$$

These relations should be valid on a particular solution of BVP with the boundary data (57). For example, choosing the so-called "soliton" profile, $N(x)=\cosh ^{-2}(x)$, i.e., $H(n)=$ $\operatorname{Arccosh}(1 / \sqrt{n})$, we have

$$
\begin{aligned}
& f=2 n(1-n) \tau_{n n}-n \tau_{n}-2 n w\left(\chi_{n}+n \chi_{n n}\right)+\left(\alpha w^{2} / 2\right) n \tau_{n n}, \\
& g=2 n(1-n) \chi_{n n}+(2-3 n) \chi_{n}+\alpha w\left(2 n \tau_{n n}+\tau_{n}\right)+\left(\alpha w^{2} / 2\right)\left(n \chi_{n n}+\chi_{n}\right) .
\end{aligned}
$$

Dependence on $\tau_{n n}$ and $\chi_{n n}$ indicates that here RGS is the second-order Lie-Bäcklund symmetry. In order to find a particular solution of a BVP (step IV), one should solve the joint system of BEs (56) and second-order ODEs that follow from the RG=FS invariance conditions (59) and (60). The resulting expressions 46 - the well-known Khokhlov solutions

$$
v=-2 \alpha n z \tanh (x-v z), \quad \alpha n^{2} z^{2}=n \cosh ^{2}(x-v z)-1,
$$

describe the process of self-focusing of a soliton beam: the sharpening of the beam intensity profile with the increase of $z$ is accompanied by the intensity growth on the beam

\footnotetext{
${ }^{13}$ In Ref.[47], where this solution was first obtained, it did not result from a regular procedure.
} 
axis. The solution (61) is valid up to the singularity point where the derivatives $v_{x}$ and $n_{x}$ tend to infinity whilst the beam intensity $n$ remains finite

$$
z_{\text {sing }}^{\text {sol }}=1 / 2 \sqrt{\alpha}, \quad n_{\text {sing }}^{\text {sol }}=2 .
$$

Here, the Lie-Bäcklund RGS enables one to reconstruct the BVP solution and describe the solution singularity for the light beam with the soliton initial intensity profile. One more example of an exact BVP solution obtained with the help of Lie-Bäcklund RGS (with the initial beam profile in the form of a "smoothed" step) can be found in [46].

For arbitrary boundary data, it turns to be impossible to fulfill the condition (59) with the help of the Lie-Bäcklund symmetries of any finite order, and one is forced to use a different algorithm 31, 46 of RGS constructing, based on the approximate group methods. Here, (step I)) $\mathcal{R} \mathcal{M}$ is given by BEs (56) with a small parameter $\alpha$, and coordinates of the group generator (58) (and, hence, coordinates of the RGS operators) appear as infinite series in powers of $\alpha$

$$
f=\sum_{i=0}^{\infty} \alpha^{i} f^{i} ; \quad g=\sum_{i=0}^{\infty} \alpha^{i} g^{i} .
$$

The procedure of finding the coefficients $f^{i}, g^{i}$ (step II)) leads to the system of recurrent relations that express higher-order coefficients $f^{i+1}, g^{i+1}$ in terms of previous ones $f^{i}, g^{i}$. It means that once the zero-order terms are specified, the other terms are reconstructed by the recurrent relations.

The coefficients $f^{i}$ and $g^{i}$ contain an arbitrary function of $n, \chi_{[s]}$ and $\tau_{[s]}-w\left(s \chi_{[s]}+\right.$ $\left.n \chi_{[s+1]}\right)$ where subscript $[s]$ denotes the partial derivative of the order $s$ with respect to $n$. This arbitrariness is eliminated by the procedure of group restriction (step III)), i.e., by imposing the invariance condition (59). For particular forms of $f^{0}$ and $g^{0}$, that is for partial boundary conditions (57), infinite series are truncated automatically, and we arrive at the exact $R G S$.

One example of this kind is given by Eqs.(60) that have a binomial structure $f=$ $f^{0}+\alpha f^{1}, g=g^{0}+\alpha g^{1}$. If we neglect the higher-order terms in the case of arbitrary boundary conditions (when series (63) are not truncated automatically), then we get an approximate $R G S$ which produces an approximate solution to the BVP. As an example, we give here two sets of expressions for the coordinates $f^{i}$ and $g^{i}$ for the Gaussian initial profile with $N(x)=\exp \left(-x^{2}\right)$, i.e., $H(n)=(\ln (1 / n))^{1 / 2}$, which define approximate RGS

$$
\begin{aligned}
& \text { a) } f^{0}=1+2 n \chi \chi_{n}, \quad g^{0}=0, \quad f^{1}=-2 \tau \tau_{n}+\frac{\tau^{2}}{n}, \quad g^{1}=-2\left(\tau \chi_{n}+\chi \tau_{n}\right), \\
& \text { b) } f^{0}=2 n\left(\tau \chi_{n}+\tau_{n} \chi\right), \quad g^{0}=1+2 n \chi \chi_{n}, \quad f^{1}=2 \chi \tau_{\alpha}, \quad g^{1}=2\left(\chi \chi_{\alpha}-\tau \tau_{n}\right) .
\end{aligned}
$$

Here, linear dependencies of $f$ and $g$ upon first-order derivatives indicate that RGS is equivalent to Lie point symmetry. The peculiarity of the case $\mathbf{b}$ is a dependence of 
$f$ and $g$ not only on derivatives with respect to $n$ but also with respect to $\alpha$ : it means that the parameter $\alpha$ is also involved in group transformations. In the non-canonical representation (33), the RGS generator in this case has the form

$$
R_{\text {Guass } 1}=2 \tau \partial_{w}+2 n \chi \partial_{n}+2 \alpha \chi \partial_{\alpha}-\partial_{\chi}
$$

The last step IV is performed in a usual way by solving the joint system of BEs (56) and equations that follow from the $\mathrm{RG}=\mathrm{FS}$ invariance condition (59), or else, using invariants of associated characteristic equations for RG operator provided that RGS is a Lie point symmetry. We give here the solution that follows from RGS (64),

$$
x^{2}=\left(1-2 \alpha n z^{2}\right)^{2} \ln \frac{1}{n\left(1-\alpha n z^{2}\right)}, \quad v=-\frac{2 x \alpha n z}{1-2 \alpha n z^{2}} .
$$

These expressions describe a self-focusing Gaussian beam (the plot $n(x)$ for this solution is presented at the end of the section on Figure 2), that is qualitatively very similar to the spatial evolution of the soliton beam (61). Moreover, the singularity position and the value of maximum beam intensity at this point coincide with analogous values (62) for the soliton beam. Although formulae (65) correspond to an approximate BVP solution, they exactly describe the behaviour of $n$ on the beam axis at $x=0$. To estimate the reliability of result (65) in the off-axis region, we compared it with another approximate BVP solution which arises from the approximate RGS in the case a. These approximations agree very well (details are presented in [46]), thus proving the accuracy ${ }^{[4]}$ of the RG approach.

\subsection{Cylindrical geometry}

In the above discussion we dealt with the plane beam geometry and took into account only effects of nonlinear beam refraction, neglecting diffraction. The flexibility of RGS algorithm allows one to apply it in a similar way to a more complicated model as compared to (56), e.g., for the cylindrical beam geometry, $\nu=2$. Omitting technical details, we present the RGS generator for the cylindrical parabolic beam with $N=1-x^{2}$

$$
R_{p a r}=\left(1-2 \alpha z^{2}\right) \partial_{z}-2 \alpha z x \partial_{x}-2 \alpha(x-v z) \partial_{v}+4 \alpha n z \partial_{n} .
$$

The BVP solution is expressed in terms of group invariants for this generator:

$$
\mathcal{J}_{1}=\frac{x^{2}}{\varrho} ; \quad \mathcal{J}_{2}=n \varrho ; \quad \mathcal{J}_{3}=2 \alpha x^{2}-v^{2} \varrho+\frac{x v}{2} \varrho_{z} ; \quad \varrho=\left(1-2 \alpha z^{2}\right) .
$$

The explicit form of dependencies of $\mathcal{J}_{2}=1-\mathcal{J}_{1}^{2}, \mathcal{J}_{3}=2 \alpha \mathcal{J}_{1}$ upon $\mathcal{J}_{1}$ follows from the boundary conditions (55). They lead to the well-known solution [47]

$$
v=(x /(2 \varrho)) \varrho_{z}, \quad n=(1 / \varrho)\left(1-\left(x^{2} / \varrho\right)\right)
$$

\footnotetext{
${ }^{14}$ One more evidence is provided by the comparison of approximate and exact BVP solution for the soliton beam performed in 46 .
} 
that describes the convergence of the beam to the singularity point $z_{\text {sing }}^{\text {par }}=1 / \sqrt{2 \alpha}$ where $\varrho=0$ and $n \rightarrow \infty$. The solution singularity is two-dimensional here: the infinite growth of beam intensity in the vicinity of the singularity $z \rightarrow z_{\text {sing }}^{\text {par }}$ is accompanied by the infinite growth of the derivative $v_{x}$ and collapsing of the beam size in the transverse direction.

The RGS algorithm based on approximate group methods can also be applied in the case when besides nonlinear refraction also diffraction effects are taken into account. Then, the first equation in (54) should be modified by adding the diffraction term

$$
-\beta \partial_{x}\left\{\left(x^{1-\nu} / \sqrt{n}\right) \partial_{x}\left(x^{\nu-1} \partial_{x} \sqrt{n}\right)\right\} .
$$

Standard calculations done in compliance with a general scheme for thus modified $\mathcal{R} \mathcal{M}$ (for details see [48) give the RGS generator for the cylindrical beam geometry $(\nu=2)$

$$
R_{\text {Gauss } 2}=\left(1+z^{2} S_{\chi \chi}\right) \partial_{z}+\left(z S_{\chi}+v z^{2} S_{\chi \chi}\right) \partial_{x}+S_{\chi} \partial_{v}-\left[n z\left(1+\frac{v z}{x}\right) S_{\chi \chi}+\frac{n z}{x} S_{\chi}\right] \partial_{n} .
$$

Here the function $S$, defined by the form of the intensity boundary distribution,

$$
S(\chi)=\alpha N(\chi)+\frac{\beta}{\chi \sqrt{N(\chi)}} \partial_{\chi}\left(\chi \partial_{\chi} \sqrt{N(\chi)}\right),
$$

contains two small parameters, $\alpha$ and $\beta$, and, as in the case $\beta=0$, there exist specific forms of boundary distribution, $N$, for which the RGS operator (68) defines exact (not approximate) symmetry valid for arbitrary values of $\alpha$ and $\beta$.

Constructing a particular BVP solution (step IV) implies the use of group invariants related to (68), and the procedure is similar to that one for the parabolic beam. For the Gaussian wave beam, $N=\exp \left(-x^{2}\right)$, the result is as follows:

$$
v(z, x)=\frac{x-\chi}{z}, \quad n(z, x)=e^{-\mu^{2}} \frac{\chi}{x} \frac{\left(\beta-\alpha e^{-\chi^{2}}\right)}{\left(\beta-\alpha e^{-\mu^{2}}\right)} .
$$

Here $\chi$ and $\mu$ are expressed in terms of $t$ and $x$ by the implicit relations

$$
\begin{aligned}
& \beta\left(\mu^{2}-\chi^{2}\right)+\alpha\left(e^{-\mu^{2}}-e^{-\chi^{2}}\right)=2 z^{2} \chi^{2}\left(\beta-\alpha e^{-\chi^{2}}\right)^{2} \\
& x=\chi\left(1+2 z^{2}\left(\beta-\alpha e^{-\chi^{2}}\right)\right) .
\end{aligned}
$$

The solution (69) describes the self-focusing of the cylindrical Gaussian beam that gives rise to the two-dimensional singularity: both the beam intensity $n$ and derivatives $v_{x}, n_{x}$ go to infinity at the point $z_{\text {sing }}^{\text {Gauss }}=1 / \sqrt{2(\alpha-\beta)}$ provided that $\alpha>\beta$. A detailed analysis of (69) and more general solutions with a parabolic form of an eikonal at $z=0$, $v(0, x)=-x / T$, is given in 48, 45. 

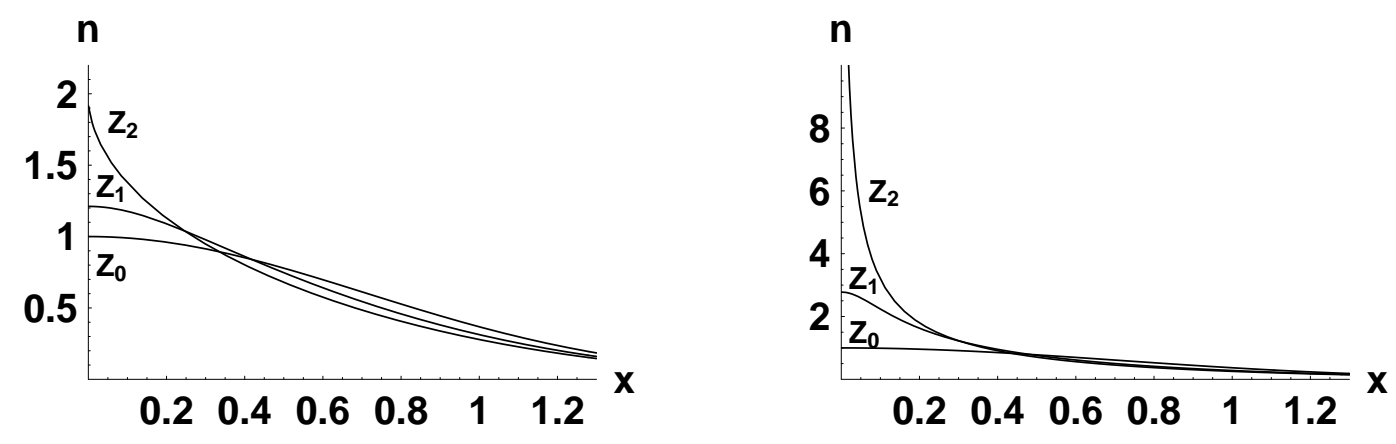

Figure 2: Intensity $n$ versus transverse coordinate $x$ for a plane (left panel) and cylindrical (right panel) beam geometry for a few values of distance $z$ from the boundary $z_{2}>z_{1}>$ $z_{0}=0$.

To illustrate the difference between the one- and two-dimensional solution singularities, in Figure 2 we present a typical behavior of the wave beam intensity, defined by Eq.(65) and (69). The left panel corresponds to the plane beam geometry, $\nu=1$, and without diffraction, $\beta=0$, while the right one is concerned with a cylindrical wave beam, $\nu=2$, with both nonlinearity and diffraction effects included. Diverse curves describe beam intensity distribution upon coordinate $x$ at different distances from the medium boundary, where we have the collimated Gaussian beam, $N=\exp \left(-x^{2}\right)$.

It is clear that in the plane geometry the derivative of the beam intensity with respect to $x$ turns to infinity at some singular point, while the value of intensity on the axis remains finite. In cylindrical case the solution singularity is two-dimensional: both the beam intensity and its derivative with respect to transverse coordinate turn to infinity simultaneously at point $z_{\text {sing }}$. This last example demonstrates the possibility of the RGS approach to analyse two-dimensional singularity. In the practice of $\mathrm{RG}$ application to critical phenomena, this correlates with the case of "two renormalization groups" 39].

\section{Overview}

To complete our review, we indicate milestones in evolution of the RG concept. Since its appearance in QFT, RG served as a powerful tool of analyzing diverse physical problems and improving solution singularities disturbed by perturbation approximation.

The development of the RG concept can be divided into two stages. The first one (since the mid-50s up to the mid-80s) is summarized in Figure 3. Besides early history (discovery of RG, formulation of the RG method and application to UV and IR asymptotics), it comprises the devising of the Kadanoff-Wilson RG in the 70s and following explosive expansion into other fields of theoretical physics.

During this stage, the formulation of the RG method was based on the unified scaling transformation of an independent variable (and/or some parameters) accompanied by a 


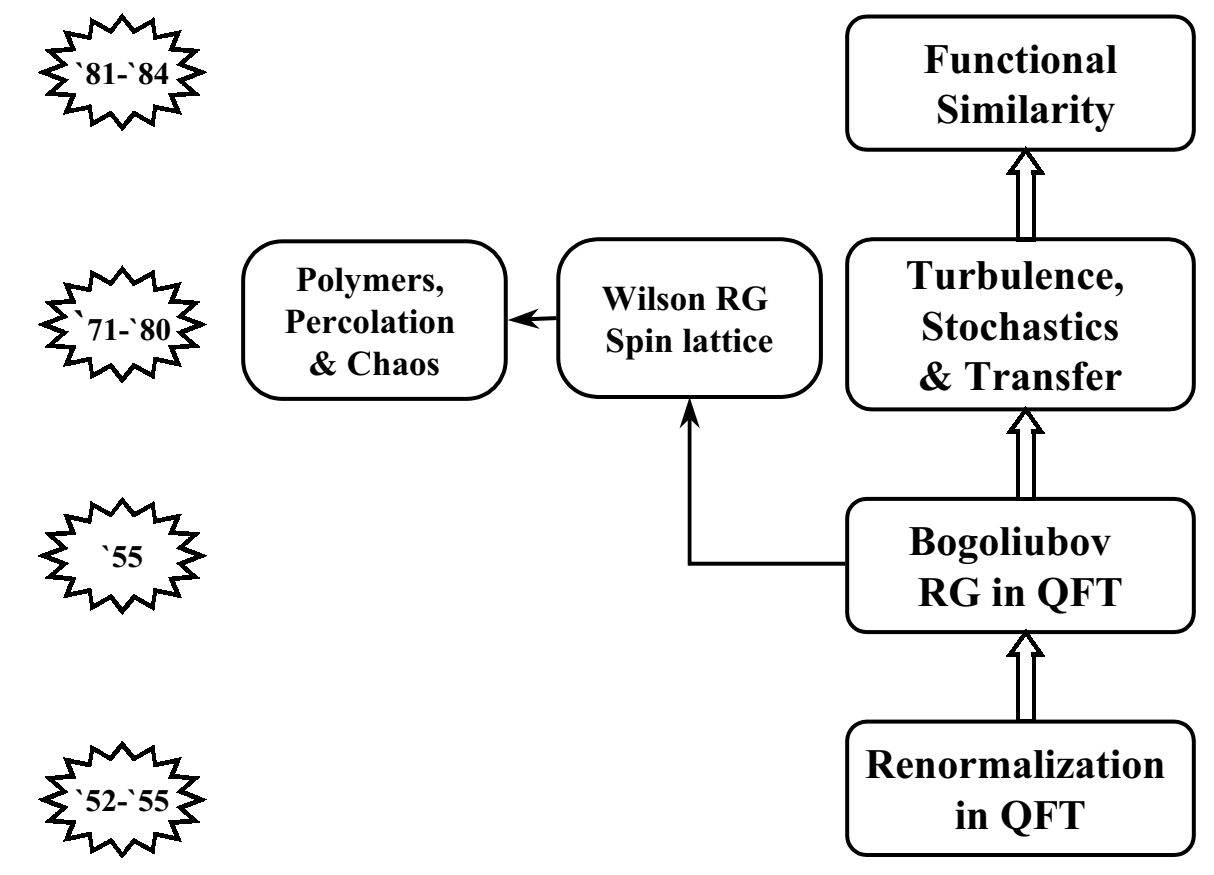

Figure 3: Early development of concept: from Bogoliubov RG to Wilson RG and FS.

more complicated transformation of a solution characteristic $g_{\mu}=\bar{g}(\mu, g)$ - see Eqs.(10), (13) and (16) in Section 1.2.

Here, the main role of $\mathrm{RG}=\mathrm{FS}$ was the a priori establishment of the fact that the solution under consideration admits functional transformations that form a group.

Any particular implementation of the RG symmetry differs in the form of the function $(\mathrm{s}) \bar{g}(\mu, g)$ (or $\beta(g))$ which, in an every partial case, is obtained from some approximate solution.

The next stage, after the mid-80s, is depicted in the Figure 4. The scheme describes the entire evolution of the Bogoliubov RG. There were several important reasons in further devising the RG concept in theoretical physics at this period. On the one hand, it was due to the extension of the notion of FS and RG symmetry that until then were based on oneparameter Lie group of point transformations. Appending multi-dimensional Lie point groups and Lie-Bäcklund groups to possible realization of group symmetry enhanced the capability of RG method. On the other hand, this additional possibility arose due to the mathematical apparatus that was used in mathematical physics to reveal RGS. The advantage came from infinitesimal transformations that enabled to describe RGS by an algebra of RG generators. However, in contrast to the situation typical of QFT models with only one operator, in mathematical physics we have finite or infinite-dimensional algebras. Both their dimension and the method of construction depend upon a model 


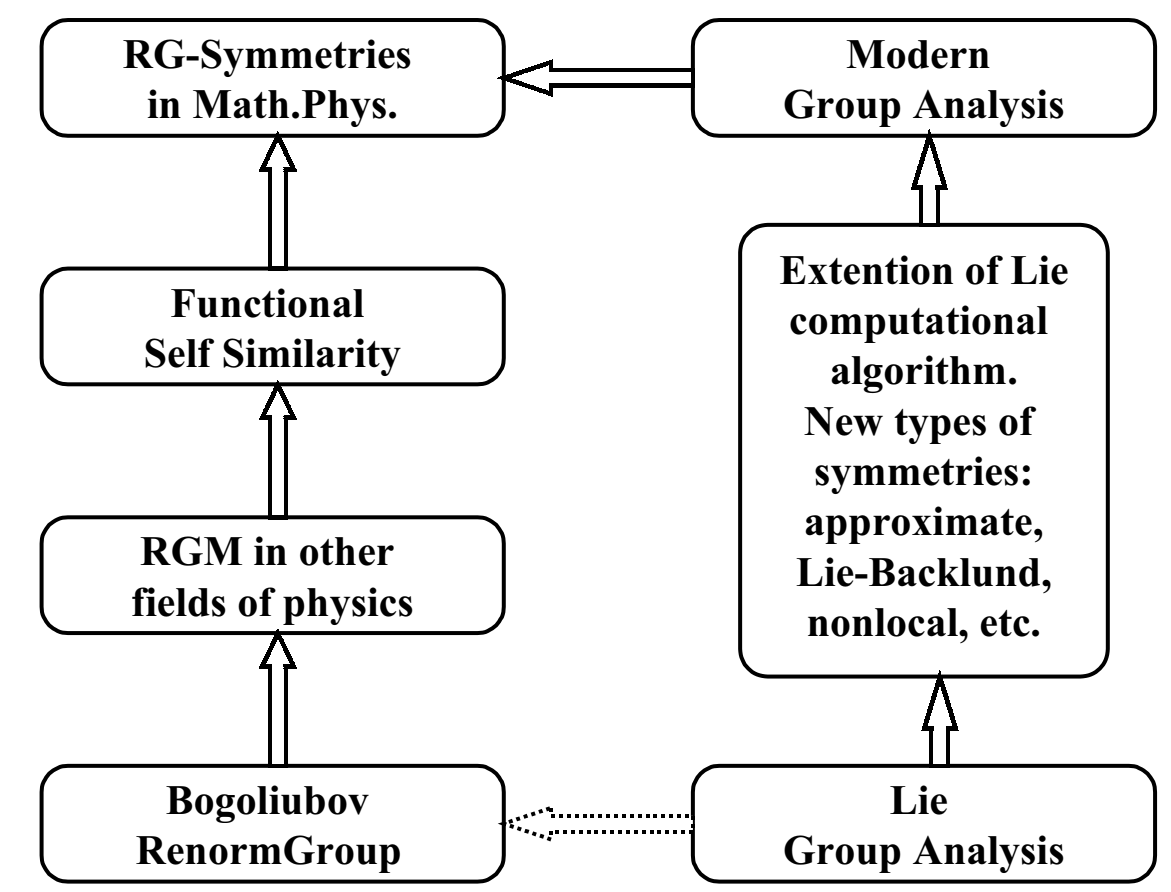

Figure 4: Evolution of concept: from the Bogoliubov RG via FS to RG-symmetries.

employed and upon a form of boundary conditions.

The use of infinitesimal approach results in constructing the RG-type symmetry with the help of regular methods of group analysis of DEs. Precisely, this regular algorithm naturally includes the $\mathrm{RG}=\mathrm{FS}$ invariance condition in the general scheme of constructing and application of RGS generators (see also our recent review 449). Within the infinitesimal approach this condition is formulated in terms of vanishing of canonical RG operator coordinates, which is especially important for Lie-Bäcklund RGS because finite transformations in this case are expressed as formal series. In particular, this property attribute a new feature to the RG analysis of a BVP solution with singular behavior, making a singularity analysis more powerful.

At the same time, as the group analysis technique is still developing - here we mean both extension to new types of symmetries and application to more complicated mathematical models, e.g., including integro-differential equations - we have a clear perspective that the possibilities of a regular scheme based upon the Bogoliubov renormalization group method are far from being exhausted.

\section{Acknowledgments}

The authors are grateful to Professors Cris Stephens and Denjoe O'Connor for invitation to participate in the Conference "Renormalization Group 2000". They are indebted to these gentlemen for useful discussions and comments. This work was partially sup- 
ported by grants of the Russian Foundation for Basic Research (RFBR projects Nos

96-15-96030, 99-01-00232 and 99-01-00091) and by INTAS grant No 96-0842, as well as by the Organizing Committee of the above-mentioned meeting.

\section{References}

[1] E.E.C. Stueckelberg and A. Petermann, Helv. Phys. Acta 22 (1953) 499-520.

[2] D.V.Shirkov, Historical Remarks on the Renormalization Group, Appendix in the collective monograph Renormalization: From Lorentz to Landau (and Beyond), Ed. Laurie M.Brown, Springer-Verlag, N.Y., 1993, 167-186; D.V.Shirkov, On the Early Days of Renormalization Group, in The Rise of the Standard Model (Proceed. 3rd Intern. Symposium on the History of Particle Physics, SLAC, 1992), Eds. Lilian Hoddeson et al., Cambridge Univ. Press. 1997, 250-258.

[3] M. Gell-Mann and F. Low, Phys. Rev. 95 (1954) 1300-1312.

[4] N.N. Bogoliubov and D.V. Shirkov, Doklady Akad. Nauk SSSR 103 (1955) 203-206 - in Russian; see also in [7].

[5] N.N. Bogoliubov and D.V. Shirkov, Doklady AN SSSR 103 (1955) 391-394 - in Russian; see also in [7].

[6] D.V. Shirkov, Doklady AN SSSR 105 (1955) 972-975 — in Russian. See also in [7].

[7] N.N. Bogoliubov and D.V. Shirkov, Nuovo Cim. 3 (1956) 845-863.

[8] L.V. Ovsyannikov, Doklady AN SSSR 109 (1956) 1112-1115 (in Russian); for English translation see In the Intermissions ... Ed. Yu. Trutnev, WS, 1998, pp 76-79; C. Callan, Phys. Rev. D 2 (1970) 1541-1547; K. Symanzik, Comm. Math. Phys. 18 (1970) 227-246.

[9] L.D. Landau et al., Nuovo Cim. Supp.3, (1955) 80-104.

[10] I.F. Ginzburg, Doklady AN SSSR 110 (1956) 535-538 - in Russian, see Chapter "Renormalization Group" in [11.

[11] N. Bogoliubov and D. Shirkov, Introduction to the Theory of Quantized Fields, 1959; 1980, Wiley-Interscience, N.Y.

[12] D.V. Shirkov, Theor. Math. Phys. 119 (1999) 55-66; hep-th/9810246.

[13] L. Kadanoff, Physics 2 (1966) 263-272.

[14] K. Wilson, Phys.Rev. B4 (1971) 3174-3183. 
[15] P.G. De Gennes, Phys. Lett. 38A (1972) 339-340; J. des Cloiseaux, J. Physique (Paris) 36 (1975) 281-292.

[16] V.I. Alkhimov, Theor. Math. Phys. 39 (1979) 422-424; 59 (1984) 591-597.

[17] T.L. Bell et al., Phys. Rev. A17 (1978) 1049-1057; G.F. Chapline, Phys. Rev. A21 (1980) 1263-1271.

[18] B.V. Chirikov, Lecture notes in physics 179(1983) 29-46; B.V. Chirikov \& D.L. Shepelansky, Chaos Border and Statistical Anomalies - in [24], 221-250; Yu. G. Sinai \& K.M. Khanin, Renormalization group method in the theory of dynamical systems - in [24], 251-277; A. Peterman and A. Zichichi, Nuovo Cimento 109A (1996) $341-355$.

[19] D.V. Shirkov, Sov. Phys. JETP 9 (1959) 421-424.

[20] C. DeDominicis and P. Martin, Phys. Rev. A19 (1979) 419-422. L.Ts. Adzhemyan et al, Theor.\& Math. Phys. 58 (1984) 47-51; 64 (1985) 777-784; A.N. Vasiliev, Quantum Field Renormalization Group in the Theory of Turbulence and in Magnetic Hydrodynamics, in [24], pp. 146-159.

[21] A.N. Vasiliev, Quantum Field Renormalization Group in the Theory of Critical Behavior and Stochastic Dynamics, PINF Publ., St-Petersburg, 1998, 773 pp - in Russian, also Gordon \& Breach, Amsterdam (in preparation).

[22] D.V. Shirkov, Teor. Mat. Fiz. 60 (1984) 778-782; The RG method and functional self-similarity in physics - in Nonlinear and turbulent processes in physics, Ed. R.Z. Sagdeev, Harwood Acad.Publ., N.Y. 1984, v.3, 1637-1647;

[23] D.V. Shirkov, Renormalization group in modern physics, in [24] pp. 1-32; Int. J. Mod. Phys. A3 (1988) 1321-1341; Several topics on renorm-group theory, in [25], pp. 1-10; "Renormalization group in different fields of theoretical physics", KEK Report 91-13 (Feb. 1992), 85 pp.

[24] Renormalization Group, (Proceed. 1986 Dubna Conference), Eds. D.V. Shirkov, D.I. Kazakov and A.A. Vladimirov, WS, Singapore, 1988.

[25] Renormalization group '91, (Proc. of Second Intern. Conf., Sept. 1991, Dubna, USSR), Eds. D.V.Shirkov \& V.B.Priezzhev, WS, Singapore, 1992,

[26] M.A. Mnatsakanyan, Soviet Phys. Doklady 27 (1982) 856-859.

[27] G. Pelletier, Plasma Phys. 24 (1980) 421-443. 
[28] Ja.B. Zel'dovich and G.I. Barenblatt, Sov. Phys. Doklady 3 (1) (1958) 44-47; see also Chap. 5 in G.I. Barenblatt, Scaling, self-similarity, and intermediate asymptotics, Cambridge Univ. Press, 1996.

[29] V.Z. Blank, V.L. Bonch-Bruevich and D.V. Shirkov, Sov. Phys. JETP 3 (1956) 845863.

[30] D.V. Shirkov, Sov. Phys. Doklady 27 (1982) 197-200.

[31] V.F. Kovalev, V.V. Pustovalov and D.V. Shirkov, J. Math. Phys. 39 (1998) 11701188; hep-th/9706056.

[32] L.V. Ovsyannikov, Group analysis of differential equations, Acad. Press, N.Y., 1982.

[33] Peter J. Olver, Applications of Lie groups to differential equations Springer-Verlag, N. Y., 1986.

[34] CRC Handbook of Lie Group Analysis of Differential Equations, Ed. N.H.Ibragimov, in 3 volumes (CRC Press, Boca Raton, Florida, USA) 1994-1996.

[35] N.H. Ibragimov, Transformation groups applied to mathematical physics, Reidel Publ. Dodrecht-Lancaster, 1985.

[36] L.I. Sedov, Similarity and Dimensional Analysis, Academic Press, New York, 1959.

[37] G. Birkhoff, Hydrodynamics, A study in Logic, Fact and Similitude, Princeton Univ. Press, 1960.

[38] V.F. Kovalev, V.V. Pustovalov, Lie Groups and their Applications 1 (1994) 104-120.

[39] C.R. Stephens, Why two renormalization groups are better than one, in Ref. [40], pp. 392-407; Int. J. Mod. Phys. 12 (1998) 1379-1396.

[40] Renormalization group '96, (Proc. of Third Intern. Conf., Aug. 1996, Dubna, Russia), Ed. D.V.Shirkov, D.I. Kazakov and V.B.Priezzhev, JINR publ., Dubna, 1997.

[41] L.-Y.Chen, N.Goldenfeld, and Y.Oono, Phys. Rev. E 54 (1996) 376-394.

[42] J. Bricmont, A. Kupiainen and G. Lin, Comm. Pure Appl. Math. 47 (1994) 893-922.

[43] T. Kunihiro, Progr.Theor.Phys. 94 (1995) 503-514.

[44] V.F. Kovalev, S.V. Krivenko and V.V. Pustovalov, The Renormalization group method based on group analysis, in Ref.[25], pp. 300-314.

[45] V.F. Kovalev, V.Yu. Bychenkov, and V.T. Tikhonchuk, Phys.Rev. A 61 (2000) No.2. 
[46] V.F. Kovalev, Theor. Math. Phys. 111 (1997) 686-702;

V.F. Kovalev, D.V. Shirkov, J. of Nonlin. Optic. Phys. \& Materials 6 (1997) 443-454.

[47] S.A. Akhmanov, R.V. Khokhlov and A.P. Sukhorukov, Sov. Phys. JETP 23 (1966) 1025-1033.

[48] V.F. Kovalev, Theor. Math. Phys. 119 (1999) 719-730.

[49] V.F. Kovalev and D.V. Shirkov, Theor. Math. Phys. 121 (1999) 1315-1322. 